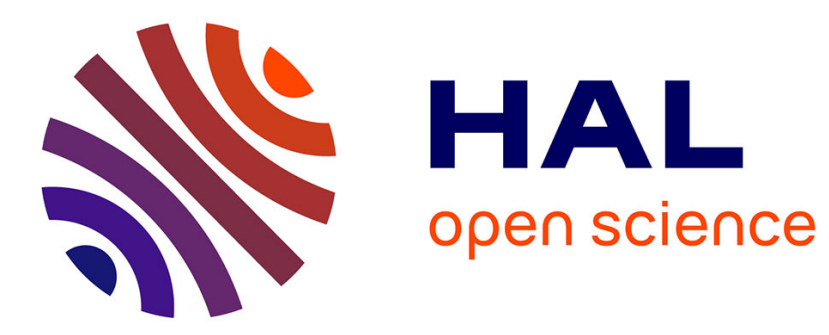

\title{
Heating of liquid foods in cans: Effects of can geometry, orientation, and food rheology
}

Rituraj Borah, Sanjay Gupta, Lubhani Mishra, Raj P Chhabra

\section{To cite this version:}

Rituraj Borah, Sanjay Gupta, Lubhani Mishra, Raj P Chhabra. Heating of liquid foods in cans: Effects of can geometry, orientation, and food rheology. Journal of Food Process Engineering, 2020, 43 (7), pp.e13420. 10.1111/jfpe.13420 . hal-03348104

\section{HAL Id: hal-03348104 \\ https://hal.science/hal-03348104}

Submitted on 17 Sep 2021

HAL is a multi-disciplinary open access archive for the deposit and dissemination of scientific research documents, whether they are published or not. The documents may come from teaching and research institutions in France or abroad, or from public or private research centers.
L'archive ouverte pluridisciplinaire HAL, est destinée au dépôt et à la diffusion de documents scientifiques de niveau recherche, publiés ou non, émanant des établissements d'enseignement et de recherche français ou étrangers, des laboratoires publics ou privés. 


\section{Journal of Food Process Engineering}

\section{HEATING OF LIQUID FOODS IN CANS: EFFECTS OF CAN GEOMETRY AND ORIENTATION, AND OF FOOD RHEOLOGY}

\begin{tabular}{|r|l|}
\hline Journal: & Journal of Food Process Engineering \\
\hline Manuscript ID & JFPE-2019-Jan-0015.R2 \\
\hline Danuscript Type: & Original Article \\
\hline Author: & 08-Aug-2019 \\
\hline & $\begin{array}{l}\text { Complete List of Authors: } \\
\text { Engingeran, Rituraj; Indian Institute of Technology Kanpur, Chemical Indian Institute of Technology Kanpur, Chemical } \\
\text { Enghra, Lubhani; Indian Institute of Technology Kanpur, Chemical } \\
\text { Engineering } \\
\text { Chhabra, Raj; Chemical Engineering; Chemical Engineering }\end{array}$ \\
\hline Keywords: & $\begin{array}{l}\text { Sterilization time, Slowest Heating Zone, Power-law model, Heat } \\
\text { penetration, Food can }\end{array}$ \\
\hline
\end{tabular}

\section{SCHOLARONE Manuscripts}




\title{
$\underline{\text { Revised manuscript (JFPE-2019-Jan-0015.R1) }}$
}

\section{HEATING OF LIQUID FOODS IN CANS: EFFECTS OF CAN GEOMETRY, ORIENTATION AND FOOD RHEOLOGY}

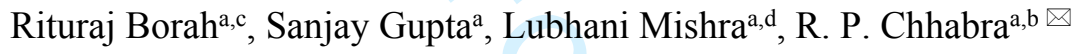 \\ aDepartment of Chemical Engineering, Indian Institute of Technology Kanpur, Kanpur, U.P., India - \\ 208016 \\ ${ }^{b}$ Department of Chemical Engineering, Indian Institute of Technology Ropar, Rupnagar, Punjab, India - \\ 140001 \\ ${ }^{\mathrm{c}}$ Department of Bioscience Engineering, Universiteit Antwerpen, Antwerp, Belgium - 2000 \\ dDepartment of Chemical Engineering, Pandit Deendayal Petroleum University, Gandhinagar, Gujarat, \\ India -382007
}

\footnotetext{
$\llbracket$ Author for correspondence: Tel: +91 512 6797393; Fax: +91 5122590104.

E-mail address: chhabra@,iitk.ac.in (R. P. Chhabra)
} 


\begin{abstract}
In this work, the effect of geometry and orientation of food cans on the heating characteristics of processed liquid foods has been investigated extensively. Elliptical and cylindrical cans of varying aspect ratios in different orientations were considered in order to delineate their effect on the heating rate, especially, the rate of increase of temperature in the Slowest Heating Zone (SHZ) is studied over wide ranges of conditions. Additionally, the effect of rheological parameter, $n$ in the simple power-law model indicating shear thinning $(n<1)$, Newtonian $(n=1)$ and shear thickening $(n>1)$ on the heating characteristics was also studied. The flow and heat transfer characteristics were analyzed with the help of velocity vectors, isotherm contours, average Nusselt number, SHZ temperature and heat penetration parameters. Comparisons were made in terms of the sterilization time and heat penetration parameters to identify the preferable geometries and orientations of food cans for effective heating of non-Newtonian food stuffs.

\section{Practical Applications}

Processed foodstuffs are produced in various forms ranging from that in solid, liquid, or as heterogeneous mixtures. Often such liquid and heterogeneous suspensions products are viscous non-Newtonian in character and their thermal processing (including pasteurisation, sterilisation, etc.) tends to be much more challenging than that of their Newtonian counterparts like air and water. This work explores heating of non-Newtonian liquid foodstuffs in cans of various shapes, geometries and in different orientations in the free convection regime. The results show that depending upon the rheological properties of the products, some orientations and/or geometries offer potential advantages in terms of shorter processing times. This information can be of great potential in customizing the design of containers for different food products as well as of different rheological properties.
\end{abstract}

Keywords: Food can, Slowest Heating Zone, Power-law model, Heat penetration, Sterilization time, Nusselt number. 


\section{Nomenclature}

$a, b: \quad$ major and minor axis of elliptical cross section, $m$

$A: \quad \quad \quad$ ratio of height to width $\left(D_{e q}\right), A=H / D_{e q}$, dimensionless

$B r: \quad \quad$ Brinkman number, $B r=\frac{\mu u_{c}^{2}}{k \Delta T}$, dimensionless

CMC : $\quad$ Carboxymethyl Cellulose

$C_{p}: \quad$ heat capacity, $\mathrm{J} / \mathrm{kg}^{\circ} \mathrm{C}$

$D_{e q}: \quad$ equivalent diameter, $D_{e q}=\sqrt{ }(4 a b), m$

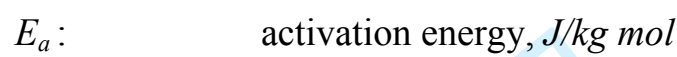

f heat transfer factor, $s$

$g$ : $\quad$ acceleration due to gravity, $\mathrm{m} / \mathrm{s}^{2}$

Gr: $\quad$ Grashof number, $G r=\frac{L^{3} \rho^{2} \beta g \Delta T}{\mu_{o}^{2}}$ dimensionless

$H \quad$ height of can, $m$

$h_{L}$ : local heat transfer coefficient, $W m^{-2}{ }^{\circ} C^{-1}$

$j \quad$ lag factor, $s$

$k$ : thermal conductivity, $W / m{ }^{\circ} \mathrm{C}$

$L: \quad$ characteristic length, $m$

$n: \quad$ power-law index, dimensionless

$N u_{\text {avg }}$ : $\quad$ average Nusselt number, $N u_{\text {avg }}=\frac{\int_{s} N u_{L} d s}{\int_{s} d s}$, dimensionless

$N u_{L}: \quad$ Local Nusselt number, dimensionless

$p: \quad$ pressure, $\mathrm{Pa}$

$\operatorname{Pr}: \quad$ Prandtl number, $\operatorname{Pr}=\frac{C_{p} \mu_{o}}{k}$, dimensionless

$S: \quad \quad \quad$ ratio of major to minor axis of elliptical cross section, $S=\frac{a}{b}$, dimensionless

SHZ : $\quad$ slowest heating zone

$t: \quad$ time, $s$ 


$$
\begin{array}{ll}
t^{*}: & \text { time, } t^{*}=\frac{t u_{c}}{L}, \text { dimensionless } \\
T: & \text { temperature, }{ }^{\circ} \mathrm{C} \\
T_{o}: & \text { uniform initial temperature, }{ }^{\circ} \mathrm{C} \\
T_{c} & \text { temperature at the center of the can, }{ }^{\circ} \mathrm{C} \\
T_{S H Z} & \text { temperature of the slowest heating zone, }{ }^{\circ} \mathrm{C} \\
T_{w}: & \text { temperature at the walls, }{ }^{\circ} \mathrm{C} \\
<T^{V>:} & \text { volume average temperature, }<T^{V}>=\frac{\int T(x, y, z) d V}{V},{ }^{\circ} \mathrm{C} \\
\Delta T: & \text { initial temperature difference, } \Delta T=T_{w}-T_{o},{ }^{\circ} \mathrm{C} \\
U: & \text { velocity vector, } m / s \\
u_{c}: & \text { characteristic velocity, } u_{c}=\sqrt{L g \beta \Delta T}, m / s \\
x, y, z: & \text { spatial coordinates, } m \\
x^{*}, y^{*}, z^{*}: & \text { spatial coordinates, dimensionless }
\end{array}
$$

\section{Greek Symbols}

$\alpha: \quad$ angle of inclination, degree $\left({ }^{\circ}\right)$

$\beta: \quad$ coefficient of volumetric thermal expansion, $\beta=-\frac{1}{\rho_{o}}\left(\frac{\partial \rho}{\partial T}\right)_{p},{ }^{\circ} C^{-1}$

$\dot{\gamma}: \quad$ shear rate, $s^{-1}$

$\dot{\gamma}^{*}: \quad$ shear rate, $\dot{\gamma}^{*}=\frac{\dot{\gamma} L_{c}}{u_{c}}$, dimensionless

$\zeta: \quad$ activation energy, $\varsigma=\frac{E_{a}\left(\mathrm{~T}_{w}-T_{o}\right)}{R T_{w} T_{o}}$, dimensionless

$\theta: \quad$ temperature, $\theta=\frac{T-T_{o}}{T_{w}-T_{o}}$, dimensionless

$\theta_{S H Z}: \quad S H Z$ temperature, $\theta=\frac{T_{S H Z}-T_{o}}{T_{w}-T_{o}}$

$<\theta^{V}>$ : $\quad$ volume average temperature, $<\theta^{V}>=\frac{\int \theta(x, y, z) d V}{V}$, dimensionless

$\kappa: \quad$ isothermal compressibility, $\kappa=-\frac{1}{V}\left(\frac{\partial V}{\partial p}\right)_{T}, P a^{-1}$

$\mu: \quad$ viscosity, $P a . s$

$\mu_{o}: \quad$ zero shear viscosity, $P a . s$ 


$$
\begin{array}{ll}
<\mu^{V>}: & \text { volume average viscosity, }<\mu^{V}>=\frac{\int \mu(x, y, z) d V}{V}, P a . s \\
\rho: & \text { density, } \mathrm{kg} / \mathrm{m}^{3} \\
\rho_{o}: & \text { reference density, } \mathrm{kg} / \mathrm{m}^{3} \\
\tau: & \text { extra stress tensor, } \mathrm{Pa}
\end{array}
$$

\section{INTRODUCTION}

Heat transfer during thermal treatment of liquid canned food takes place primarily by free convection and conduction due to the confined nature of fluid (Hiddink, 1975; Al-Baali \& Farid, 2006). In the free convection regime, the geometry of the can has significant effect on the resulting heat transfer characteristics. Consequently, much research effort has been directed at studying heating of liquid foods in containers during thermal treatment processes such as sterilization, pasteurization, etc. (Simpson, 2009; Rao \& Anantheswaran, 1988).

The early experimental and theoretical studies were devoted towards understanding and improvement of the thermal processing of packaged foods were more successful for solid foods such as asparagus, corn, cherry, strawberry, etc., because only the conduction equation was employed to model heat transfer (Thompson, 1919; Hayakawa \& Ball, 1971; Gillespy, 1951, 1953). On the other hand, heat transfer in liquid foods filled in cans is complex due to the mixed mode of heat transfer, namely, conduction and free convection. Thus, analytical but approximate approaches such as incorporation of an effective diffusivity for solid-liquid suspensions, assumption of thermal resistance limited to the boundary layer, etc., were adopted in the early studies to empirically account for these effects (Thompson, 1919; Thorne \& Howitt, 1972, Jones, 1931; Schultz \& Olson, 1938; Evans et al., 1968). Also, the early experimental studies on the heating of liquid foods elucidated the convective flow patterns and the resultant convective component of heat transfer very well. The upward flow of the fluid near the wall, the downward flow in the central region of the can and the existence of a mobile cold temperature point explained in detail in the subsequent computational studies, etc. were reported in many of these experimental works (Jackson \& Olson, 1940; 
Tani, 1940; Fagerson \& Esselen, 1950; Blaisdell, 1963; Reichter \& Bielig, 1973; Hiddink et al., 1976; Zechman \& Pflug, 1989).

Generally, such experimental studies are not only tedious, but the use of intrusive sensing devices in such studies also introduces considerable errors and the contamination is always a concern in such applications (Marra \& Romano, 2003). Also, real-time measurements of the temperature of the mobile SHZ is still an important topic of active investigation, even for solid foods (Rinaldi et al., 2012). In the early numerical studies, although the agreement between the predicted and experimental results was not always satisfactory (Barakat \& Clark, 1966; Hiddink, 1975), it paved a way for future developments (Engelman \& Sani, 1983; Datta, 1985). Over the years, numerous investigators have studied sterilization of different fluids such as milk, beer, cherry juice, etc., using different CFD frameworks (Anand Paul et al., 2011; Abdul Ghani et al., 2001, 2002; Augusto et al., 2010). Solid-in-liquid systems where combined conduction-convection heat transfer is possible have also been studied due to their common occurrence (Rabiey et al., 2007; Kızıltaş et al., 2010). For vertical cylindrical or conical cans, simplification of the numerical analysis by the axisymmetric assumption significantly reduces the required computational effort, both in terms of time and resources without introducing significant errors (Kumar et al., 1990; Kumar \& Bhattacharya, 1991, Varma \& Kannan, 2005, 2006; Kannan \& Neeharika, 2007; Kannan \& Sandaka, 2008; Moraga et al., 2011; Lespinard and Mascheroni, 2012). A number of investigators have also explored other geometrical shapes such as horizontal cylinders, food pouches, brick shaped containers, etc. (Abdul Ghani et al., 2001; Augusto \& Cristianini, 2012) that require 3D analysis. In a recent study, Rinaldi et al. (2018) investigated circular, hexagonal, square and flared cylindrical geometries to compare heat transfer characteristics in the context of thermal sterilization processes. However, it is not yet possible to completely delineate the role of can geometry or of the thermo-physical properties of the model food systems in these applications.

It is thus fair to say that little is known about the effect of different geometrical aspects of the food cans on the heating characteristics of non-Newtonian liquid foods during their thermal processing. The present study aims to fill this gap in the literature in cans of different geometry and orientation. The transient 
continuity, momentum and the energy equation were solved numerically to simulate the development of flow and heat transfer characteristics of the process.

\section{DETAILS OF CAN GEOMETRY AND FLUID PROPERTIES}

The volume of a standard can (Kannan \& Sandaka, 2008) was held constant for all geometries and orientations considered in this work so that the effects of the pertinent parameters can be elucidated in an unambiguous manner. As shown in Figure 1, three geometric parameters $A, S$ and $\alpha$ completely define a configuration. $A$ is the ratio of height, $H$ to the equivalent diameter, $D_{e q}$, while $S$ represents the aspect ratio of the horizontal cross section of a can in upright position, which is the ratio of the major and minor axes of an ellipse, i.e., $S=1$ corresponds to a circular can geometry. The angle of inclination from vertical is defined by $\alpha$ as shown in Figure 1. It is appropriate to add here that it is common to use hydraulic diameter for non-circular cross-sections for the purpose of estimating pressure drops. It is easily seen that the equivalent diameter, $D_{\text {eq }}$, and hydraulic diameter $D_{h}$ of an elliptical cross-section (semi-major axis $a$ and ratio, $S)$ are interrelated. For this case, $D_{e q}=2 b \sqrt{S}$ and $D_{h}=2 \sqrt{2}\left(S b / \sqrt{1+S^{2}}\right)$. Evidently, for $S=1$, the two values are identical whereas the ratio $D_{e q} / D_{h}=(1 / S)\left(\sqrt{S\left(1+S^{2}\right) / 2}\right)$, is 1.12 for both $S=2$ and $S$ $=0.5$. The two values thus only differ from each other by a constant factor and one can easily convert the results from one format to another. Similarly, it is possible to scale $S$ also with $a$, but as seen above, one can easily recast the present results in any other such format.

A model liquid food $0.85 \% \mathrm{w} / \mathrm{w}$ aqueous Carboxymethyl Cellulose solution has been used in this study in line with the previous studies (Kumar \& Bhattacharya, 1991; Kannan \& Neeharika, 2007; Varma \& Kannan, 2005, 2006); the corresponding thermo-physical properties of the model fluid are summarized in Table 1. The viscosity-shear rate relationship of the model fluid is approximated by the truncated powerlaw model (Bird et al., 1987) in which the power-law index $n$ denotes the shear rate dependence of the fluid viscosity as follows: 
$\mu=K_{o} \exp \left(\frac{E_{a}}{R T}\right) \dot{\gamma}^{(n-1)}$

Steffe (1996) lists a number of food products such as carrot puree, tomato puree, applesauce, apricot puree, banana puree, etc., that exhibit thermo-physical properties similar to the model shear-thinning fluid used here, with the value of $n$ as low as 0.2 . Although shear-thickening behavior is not encountered as frequently as shear-thinning, certain food systems like starch pastes, mixtures of hydrocolloids and starches, etc., do exhibit the value of $n$ as high as 1.96 (Steffe, 1996). The temperature-dependence of the viscosity is defined by an Arrhenius like relationship between the power law consistency and temperature, eq. (1). The values of these parameter for the model food are also summarized in Table 1.

\section{PROBLEM DEFINITION AND GOVERNING EQUATIONS:}

The problem studied herein is formulated in terms of the process conditions of a typical sterilization carried out in a still retort. A constant temperature $T_{w}=121^{\circ} \mathrm{C}$ is assumed on all walls of the food-cans, while a uniform initial fluid temperature $T_{o}=40^{\circ} C$ is assumed for the fluid at rest at $t=0$ in the can. No-slip boundary condition is prescribed on solid walls of the food can. The thermal conductivity $(k)$ and specific heat $\left(C_{p}\right)$ of the model food are assumed to be temperature-independent. In fact, the fluid density is also assumed to be constant, except for the density term appearing in the buoyancy term in the momentum equation. Viscous dissipation is also neglected as the fluid motion can be expected to be weak in free convection since the maximum value of the $B r$ is $\sim 0.004$. Due to the absence of any heat sink within the domain, the flow field is inherently transient which will never reach a steady state. The time dependent forms of the field equations for an incompressible fluid are written as follows:

Continuity: $\nabla \cdot U=0$

Momentum: $\quad \rho\left(\frac{\partial U}{\partial t}+U \cdot \nabla U\right)=-\nabla p+\nabla \cdot \tau+\rho g \beta\left(T-T_{0}\right) \delta_{i k}$

Energy: $\quad \frac{\partial T}{\partial t}+U \cdot \nabla T=\frac{k}{\rho C_{p}} \nabla^{2} T$ 
The familiar Boussinesq approximation (Tritton, 1977) is used here to capture the variation of the fluid density with temperature as,

$\rho_{o}-\rho=\rho_{o} \beta\left(T-T_{o}\right)$

where, $\beta$ is the coefficient of thermal expansion and $\rho_{o}$ is the reference density corresponding to the reference temperature $T_{o}$. It is useful to recall the other conditions required for the applicability of the Boussinesq approximation (Tritton, 1977):

$$
\begin{aligned}
& \beta \Delta T<<1 \\
& g \rho \kappa L<<1 \\
& \frac{g \beta L}{C_{p}}<<1 \\
& \frac{g \beta L T_{o}}{C_{p} \Delta T}<1
\end{aligned}
$$

Finally, the extra stress tensor $\tau$ appearing in eq. (3) is expressed in terms of the viscosity (eq. 1) multiplied by the rate of strain tensor, $\dot{\gamma}$, i.e., $\tau=\mu \dot{\gamma}$ (Bird et al., 1987).

In order to enhance the utility of the present results, the field equations have been rendered dimensionless using suitable scaling parameters. A characteristic velocity $u_{c}=\sqrt{\operatorname{Lg} \beta \Delta T}$, characteristic time, $t_{c}=L / u_{c}$ and the characteristic length as $L=V^{1 / 3}$ have been used to scale velocity, time and linear dimensions respectively, unless mentioned otherwise. The extra stress tensor, $\tau$ has been scaled with $\mu_{o}$ $\left(u_{c} / L\right)$. Similarly, the fluid temperature is scaled as $\theta=\frac{T-T_{o}}{T_{w}-T_{o}}$. Dimensional considerations of this configuration suggest that the flow and heat transfer characteristics to be governed by the following parameters $G r$ and $\operatorname{Pr}$ defined as follows: 
$G r=\frac{L^{3} \rho^{2} \beta g \Delta T}{\mu_{o}^{2}}$

$\operatorname{Pr}=\frac{C_{p} \mu_{o}}{k}$

where, $\mu_{o}$ is the viscosity of the fluid when the fluid is at rest at $t^{*}=0$ and $\Delta T=T_{w}-T_{0}$, i.e. the temperature driving force in the entire domain at $t^{*}=0$. It is worth noting here that $\mu_{o}$ is often termed as zero-shear viscosity as given in Table 1 and this value varies from fluid to fluid. For the present case, this value can be calculated from equation 1 with a value of minimum shear rate $0.01 \mathrm{~s}^{-1}$ and initial temperature $T_{o}=40^{\circ} C$. More detailed discussions on the truncated power law model can be found in Bird et al. (1987). In addition, the geometry (size, shape and orientation) of the food can together with the power law index, $n$ of the fluid also influences the heating characteristics of the food product. Similarly, the linearization of the exponential relationship between the viscosity and temperature yields another dimensionless group which scales the activation energy of the fluid as:

$\zeta=\frac{E_{a}\left(T_{w}-T_{o}\right)}{R T_{w} T_{o}}$

This definition of $\zeta$ evolves naturally during the course of the non-dimensionalization of the equations and linearization of the exponential term. Interestingly, it suggests the use of harmonic mean of the two temperatures as opposed to their arithmetic mean. Thus, the rate of heating of a foodstuff contained in the can is influenced by a large number of variables. The overall heating phenomena is captured in terms of the familiar Nusselt number which is defined as:

$$
N u_{L}=\frac{h_{L} L}{k}=-\frac{1}{\left(T_{w}-<T^{V}>\right)} \frac{\partial T}{\partial x^{*}}
$$




\section{NUMERICAL METHODOLOGY AND CHOICE OF NUMERICAL PARAMETERS}

The aforementioned field equations subjected to the given boundary conditions have been solved numerically using the finite element based solver COMSOL Multiphysics (version 5.2). A 3D, transient laminar flow module was used along with the module for heat transfer in fluids for the solution of the coupled equations. The partial differential equations discretized by the finite element method (FEM) result in a sparse linear system. The linear direct solver PARDISO (parallel sparse linear direct solver) in COMSOL Multiphysics was employed to solve the system of equations. PARDISO supports a wide range of sparse matrix types (e.g. real or complex, symmetric, structurally symmetric or non-symmetric) and computes the solution of such sparse linear systems of equations of the form, $\mathrm{AX}=\mathrm{B}$, on a shared or distributed memory architecture. Due to the coupling of the momentum and energy equations in free convection, the momentum and energy equation were simultaneously solved by the numerical scheme to obtain the velocity and temperature fields. More detailed discussion is available in the COMSOL Multiphysics Release Notes (www.comsol.com/support).

A grid independence study was carried out for each geometry (i.e., value of $A, S$ and $\alpha$ ) to construct an optimal computational mesh that leads to acceptable levels of accuracy of the results without necessitating 
excessive computational resources. For all the computations, a $3 D$ mesh with tetrahedral grid elements were used. The initial numerical experiments with the prismatic and hexahedral grid elements revealed their inadequacy for elliptical shaped food cans. Figure 2 shows a comparison in terms of the local Nusselt number obtained from two grids $G 1$ and $G 2$ for the model food CMC in elliptical-shaped cans. The two predictions are seen to differ from each other by less than $<1 \%$, albeit $G 2$ consists of three times as many nodes as $G 1$. Thus, $G 1$ has been used here to obtain the results reported in this work. A numerical solution of the transient equations also requires an optimum resolution of the temporal evolution of the flow and heat transfer characteristics. Hence, a time-step independence test was carried out by implementing different implicit time stepping schemes. The BDF (backward difference formulae) time stepping scheme was found to predict each time step according to the dynamics of the system in order to optimize the computation. Obviously, the initial time steps in BDF are very small (of the order of $1 \mathrm{~ms}$ ), which gradually becomes larger as the system response becomes sluggish (time rate change drops). Generalized alpha time stepping scheme is a more robust in so far that it does not allow time steps larger than a specified time step, $\Delta t$. Figure 3 compares the influence of the value of $\Delta t$ on the results suggesting that the three schemes used here yield essentially identical results. Note that due to the rather large values of time ( $\sim 500-2000 \mathrm{~s})$ needed, the results obtained with $\Delta t=0.05$ and $\Delta t=0.1 \mathrm{~s}$ are virtually indistinguishable from each other in Figures 3 and 4.Hence, the robust generalized alpha scheme with $\Delta t=0.1 \mathrm{~s}$ was used to obtain the present results. Furthermore, for prechosen values of the dimensionless parameters, the corresponding dimensions and/or the actual values of the thermophysical properties (like thermal conductivity, heat capacity, etc. ), etc. were back calculated and these were used as inputs. This is so because COMSOL solves the system of equations for a given geometry. But on the other hand, the specific values of the can size, shape, initial and wall temperatures, physical properties, etc. used here are of no particular significance and the results presented herein are valid as long as the values of the dimensionless parameter are in the range spanned here. 


\section{RESULTS AND DISCUSSION}

New extensive results on transient heat transfer to a model liquid food filled in a sealed can (of different geometries and orientations) but of a fixed volume are reported here. The present results span the values of the dimensionless groups as $G r=0.445, \operatorname{Pr}=79481, n=0.57,0.5 \leq A \leq 2,0.5 \leq S \leq 2$ and $0^{\circ} \leq \alpha \leq 90^{\circ}$. In order to delineate the influence of shape and orientation of food can in an unambiguous manner, results reported herein relate to the same food product. The flow and temperature fields are visualized in terms of the velocity vectors, velocity magnitude contours and isotherm contours. The temporal evolution of the average Nusselt number provides further insights into the overall heating characteristics. The temperature of the slowest heating zone (SHZ), which in this study is defined as the temperature of the coldest point in the food can, is obviously an important aspect in such operations aimed at complete sterilization the product. If the sterilization process is carried out only till the point when the slowest heating zone temperature just reaches the threshold bacterial deactivation temperature, these regions have the possibility of hosting some active bacteria. Naturally, the specifics of such threshold levels are strongly dependent on the type of bacteria and the other conditions and environment and hence, it will vary from one product to another and well as from one application to another. Hence, it is thus a part of problem definition. Notwithstanding this, thus, reliable knowledge of the location and the size of the slowest heating zone is important from a quality control and product homogeneity perspective. Prior to undertaking a detailed discussion of the new results obtained in this work, it is desirable to ascertain the reliability and accuracy of the numerical procedure used here by reproducing some of the results available in the literature, as discussed in the next section.

\subsection{Validation}


Prior studies on transient heat transfer to liquid foods in closed containers are limited in the open literature, though, a few studies have been reported on the heating of liquid foods in standard cans (Kannan \& Sandaka, 2008; Varma \& Kannan, 2005, 2006; Kumar \& Bhattacharya, 1991). Figure 4 shows comparisons between the present results with the above mentioned studies for a vertical cylindrical can subjected to initial and boundary conditions similar to that used in this study. Kannan and Sandaka (2008) have reported their results for a cylindrical can (A "regular" can as per the terminology used in the paper) of diameter 81 $\mathrm{mm}$ and height $111 \mathrm{~mm}$ in the vertical orientation. Thus, based on the terminology used in this study, the above-cited dimensions translate to $A=1.37, S=1, \alpha=0^{\circ}$ where $A$ is aspect ratio, $S$ is aspect ratio of the cross section (for cylinders, $S=1$ ) and $\alpha$ is the inclination angle (see Figure 1). An excellent agreement is seen to exist in most cases and the fact that the 3-D predictions are in line with the axisymmetric results confirms the flow regime indeed to be axisymmetric under these conditions. Furthermore, it is worth mentioning here that Kannan \& Sandaka, (2008) also show satisfactory agreement between their numerical results and experimental results thereby suggesting the present results to be consistent with the scant experimental results.

\subsection{Velocity vectors and velocity magnitude contours}

Figures 5 and 6 shows dimensionless velocity vectors along with the velocity magnitude contours on two perpendicular cut planes across the food cans for $A=0.5$ and $A=1.5$ respectively. For this purpose, the two (horizontal and vertical) cut planes have been selected so that the cylinder is cut into two equal halves, either from the middle or along the axis. These cut planes represent velocity magnitude and temperature variation at central planes of the cylinder which indicate the spatial distribution of velocity and temperature in the domain. The velocity vectors are shown to provide a three dimensional visualization of the flow while the cut planes provide a clearer picture than the velocity magnitudes. In $3 \mathrm{D}$ representation, the velocity vectors indicate the magnitudes rather poorly. It is often difficult to differentiate among vectors at different distances. For each value of $A$, cylindrical $(S=1)$ and elliptical $(S=0.5)$ configurations are shown in (a) and (b) parts of these figures. The evolution of the velocity field with time in each can is compared for three 
orientations. Owing to the transient nature of the flow, these plots are shown at three time instants to show the gradual temporal development of the velocity field. An overall inspection of these figures suggests that at the time instant $t^{*}=41.5$, the velocity field is very weak, as the fluid needs to be mobilized from its rest state by the feeble buoyancy induced flow and because initially, heat transfer occurs by conduction. As the temperature gradient paves the way for the establishment of density gradient thereby setting the fluid in motion due to buoyancy forces, the velocity field gradually develops. After sufficiently long time, $t^{*}=$ 207.4, the velocity vectors and their magnitude contours indicate the presence of significant fluid motion. On the other hand, as the temperature of the fluid increases, the driving force for heat transfer decreases with time. As a result, the velocity currents again become weaker (as seen in these figures at $t^{*}=414.9$ ) and eventually slowly diminish as the fluid approaches the wall temperature.

Similarly, Figure 5, the velocity field in a "short" can, i.e., $A=0.5$ in the vertical orientation indicates well developed re-circulatory velocity currents at $t^{*}=207.4$ with the fluid adjacent to the wall going up, after striking the top cold wall it descends in the central region. As the height of the can increases, the fluid has a longer residence time and the rising plume expands radially accompanied by a gradual decrease in its upward velocity. In Figure 6 for $A=1.5$ ("tall" can) in vertical orientation, this re-circulatory flow is not seen to be as strong as in the case of $A=0.5$ as the narrow shape restricts the upward velocity currents forcing a fluid element to take a circular path to flow in the downward direction. However, for $A=1.5$ and $S=0.5$ in the vertical orientation (Figure 6(b)), due to the elliptical cross section, stronger re-circulatory current can develop along the major axis. Since the "short" geometry offers room for the formation of welldeveloped recirculation patterns with a degree of ease, this may, in turn, enhance the heat transfer rate. In the inclined orientation (Figures 5 and 6), the flow patterns appear to be more complex than that in the case of vertical orientation. However, partial inclination does not seem to promote significantly the degree of convection as the fluid near the walls still has to travel along the length of the can and hence the bulk of the discussion here pertains to the vertical and horizontal orientations. In the horizontal orientation $\left(\alpha=90^{0}\right)$, the flow path in the vertical direction is short and the curvature of the shape of the can steers the moving 
fluid to take a curved part along the walls. Moreover, for $S=0.5$ in the horizontal orientation (Figures 5 and 6), the vertical dimension is further reduced. Thus, in horizontal orientation, there is a strong recirculation of the fluid which may, in turn, also lead to improved heat transfer.

\subsection{Isotherm contours}

Figures 7 and 8 show a typical development of the temperature field with time in terms of isotherm contours for $A=0.5$ and $A=1.5$ respectively. The presentation scheme here is similar to that used in Figures 5 and 6. In line with the velocity field results seen in Figures 5 and 6 , isotherm contours at $t^{*}=41.5$ indicate that, heat penetrates only up to a small distance from the walls. Hence, the isotherm contours lie along the can walls taking the shape of the can which also suggests that the mode of heat transfer is mainly conduction. This is consistent with the near absence of fluid motion at $t^{*}=41.4$ seen in Figures 5 and 6 . After sufficient time, when the velocity field is strong at $t^{*}=207.4$, the distorted shape of the isotherm contours indicates strong convective heat transfer. Thus, with the passage of time, the fluid is gradually heated by drawing varying contributions from conduction and convection. The isotherm contours shown in Figures 7 and 8 at $t^{*}=829.8$ also indicate the shape, size and location of the slowest heating zone.

\subsection{Average Nusselt number}

Figure 9 shows typical results on the average Nusselt number corresponding to different walls of the cans in terms of its evolution with time for three orientations $\alpha=0^{\circ}, \alpha=45^{\circ}$ and $\alpha=90^{\circ}$. The initial steep drop in the value of the Nusselt number is due to the onset of conduction during the initial phase and the $\Delta T$ is maximum at this point in time. With the passage of time, as the convection increases, the Nusselt number tends to increase for a while and then eventually decreases again due to the decreasing driving force for heat transfer. For a vertical can, the peak in the average Nusselt number for the bottom flat surface can be explained by the fact that the entire mass of fluid partakes in heat transfer. This also indicates that the relative contribution of the bottom surface to the overall heat transfer might increase with the decreasing value of $A$ in this orientation. On the other hand, the average Nusselt number for the top surface remains 
almost constant with time after the initial steep decrease due to conduction heat transfer. While for the lateral surface, the average Nusselt number stays very close to the average value of all the walls indicating its moderate overall contribution. For an inclined can at $\alpha=45^{\circ}$, the average Nusselt number for the top and the lateral surface is influenced very little from the value at $\alpha=0^{\circ}$. For the horizontal orientation, the average Nusselt number for the bottom half of the curved surface follows a trend similar to that for the bottom flat surface of the vertical can. Although, a well-developed re-circulatory motion region is formed for the horizontal orientation, Figure 6, but it does not seem to enhance the average Nusselt number significantly; however, this may contribute greatly to a more uniform distribution of heat in the can, especially in the colder regions.

In order to enhance the utility of the present numerical results in the context of different process conditions, it is useful to consolidate the numerical values of the average Nusselt number as a function of time. Kannan and Sandaka (2008) divided the overall heat transfer process into subcritical and supercritical regimes, and presented separate correlation for each regime. In this work, the average Nusselt number values vs time data are well approximated by the following simple empirical correlation that incorporates the exponential decay and the "Gaussian" like increase during the convection dominated period:

$$
N u=a \exp (-b t)+c \exp \left(d t^{2}\right)+e
$$

The values of the empirical constants $a, b, c, d$ and $e$ appearing in eq. 15 as well as the resulting percentage average and maximum errors are given in Table 2. The degree of fit for each value of $\alpha$ is seen to be satisfactory reflected by rather small values of the maximum \% deviation. For a given system which is similar to the one studied here, one can use Eq. 15 to estimate the value of the Nusselt number at any instant of time and by comparing it with the corresponding conduction limit, it is possible to infer indirectly the relative contributions of conduction and convection to the overall heat transfer.

\subsection{Slowest heating zone temperature}


As discussed earlier, the slowest heating zone (SHZ) temperature is particularly important in sterilization processes. It is also worthwhile to point out here that a seemingly small temperature difference (e.g., $5{ }^{\circ} \mathrm{C}$ ) may be significant in food sterilization operations as the sensory and nutritional properties of food are highly sensitive to temperature. For the simple geometries considered in this work, SHZs are not located at multiple locations (Figures 7 and 8). Figure 10 shows the combined effects of $A, S$ and $\alpha$ on the evolution of SHZ temperature with time. For the vertical orientation, since $S=0.5$ and $S=2$ are equivalent, comparisons are shown only between $S=1$ and 0.5 . In all cases, during the initial conduction dominated phase, heat penetration is limited to a thin layer of the fluid adjacent to the wall and it does not reach all the way to the core of the can. Thus, the SHZ remains at the initial fluid temperature for a certain period called lag-period. However, once the convection currents strengthen, the slowest heating zone starts to get heated rather quickly. This is also indicated by the isotherm contours shown in Figures 7 and 8 . Thus, the rate of increase of SHZ temperature depends upon both the lag-period and the heating rate after the lag-period. Both the lag period and the heating rate after the lag period depend on the development of the convective currents. The results shown in Figure 10 suggest that the heating rate increases with the decreasing value of $S$. It is the ease offered by the elliptical geometry for the development of stronger convection in all orientations.

\subsection{Sterilization time: Effect of geometry and orientation}

The food in a can is assumed to be sterilized when the SHZ temperature reaches the prescribed threshold bacterial deactivation temperature. From a process economics point of view, it is desired to have as short sterilization time as possible. A simple definition of the sterilization time is the time required for the SHZ temperature to reach the threshold temperature everywhere in the can and this may of course vary from one case to another. For mild thermal processes such as pasteurization, this temperature may be low. The threshold temperature is assumed to be $373.17 \mathrm{~K}$ (i.e., $\left.\theta_{S H Z}=0.74\right)$ in this study (Varma $\&$ Kannan, 2005). Thus, it is clear that the variation in the sterilization time as per this definition may be due to the variation in the lag period or the heating rate after the lag period or the both. 
Figure 11 (a) shows the effect of inclination on the sterilization time for cans of three aspect ratios, $A=0.5$ (short), $A=1.0$ (medium) and $A=1.5$ (tall). Evidently, as the "short" can is inclined gradually from its vertical orientation $\left(\alpha=0^{\circ}\right)$, sterilization time increases and then decreases. For the horizontal orientation ( $\alpha=90^{\circ}$ ), the sterilization time is longer than that for the vertical orientation. Conversely, as the "tall" can is inclined gradually, the sterilization time initially remains nearly unaffected. However, beyond $\alpha=45^{\circ}$, sterilization time starts to drop and eventually reaches its minimum value in the horizontal orientation of the can. Similarly, in the case of a "medium" can, sterilization time increases slightly and then starts to drop at $\alpha=45^{\circ}$ to reach the lowest value at the horizontal orientation. Thus, it is not useful to incline a "short" can during sterilization. While a "tall can" should rather be placed in the horizontal orientation in order to minimize the sterilization time.

Figure 10 clearly shows that the effect of $S$ is more pronounced in the horizontal orientation. Therefore, the effect of $S$ on the sterilization time for horizontal can of three aspect ratios, $A=0.5$ (short), $A=1.0$ (medium) and $A=1.5$ (tall) is shown in Figure 11 (b). For three values of $A$, sterilization time is seen to be minimum for a non-circular cross section can of $S=0.5$.

Figure 11 (c) shows the effect of $A$ on sterilization time for cylindrical cans $(S=1)$ in vertical and horizontal orientations. Clearly, for the vertical orientation, the sterilization time is minimum for $A=0.5$ ("short" can). As the value of $A$ increases, sterilization time also increases and it becomes constant beyond a value (i.e. $A$ $=1.75)$. However, in the horizontal orientation, the opposite trend occurs. With increasing $A$, sterilization time starts to fall after $A=0.75$ and it continually decreases till $A=2$.

Since food processing engineers are particularly interested in reducing sterilization time, a discussion about the $\%$ reduction would bring out the practical utility of these results. For instance, a "very tall" cylindrical can $(A=2$ and $S=1)$ requires the longest time for sterilization in vertical orientation. However, it drops by $15.8 \%$ in a horizontal orientation. If the can is now made elliptical with $S=0.5$ in horizontal orientation, the sterilization time is reduced to become $21.3 \%$ of that for the case $A=2$ and $S=1$ in vertical orientation. 
Similarly, changing the orientation of a "tall" cylindrical can $(A=1.5)$ from the vertical $(\alpha=0)$ to the horizontal $\left(\alpha=90^{\circ}\right)$ reduces the sterilization time by $11.3 \%$. For a medium cylindrical can $(A=1)$, sterilization time is reduced by $4.9 \%$ when the orientation is changed from vertical to horizontal and further overall reduction is $11.9 \%$ when the value of $S$ is changed from 1 to 0.5 . For a short cylindrical can $(A=$ $0.5, S=1)$, the best orientation is vertical. Thus, if the orientation of a cylindrical can is fixed as vertical, sterilization time can be reduced by $13 \%$ by changing the values of $A$ from 2 to 0.5 . Table 3 (a) to 3 (c) summarizes the findings in terms of dimensionless sterilization time.

\subsection{Heat penetration plots and parameters}

Since sterilization time is essentially a single point description, it does not reveal much details about the temperature-time history of the process. In order to develop some further insights, it is useful to introduce a statistical measure that takes into account the entire heating process. In heat penetration studies, the timetemperature data, such as that shown in Figure 10 is conventionally presented on a semi-log plot of the difference between the constant wall temperature and the variable slowest heating zone temperature in a dimensionless form $\left.\left(T_{w}-T_{S H Z}\right) /\left(T_{w}-T_{o}\right)\right)$ against the dimensionless time. Such plots are characterized by an initial constant value followed by a decreasing trend, as can be seen in Figure 12 .

This format is advantageous as part of the results shown in Figure 12 can be approximated by a linear equation and it is possible to obtain two parameters $f$-value (heat penetration factor) and $j$ - value (lagfactor) from the slope and the intercept of the curve respectively (Bigelow et al. 1920; Holdsworth \& Simpson, 2007). The heat penetration factor, $f$-value denotes the time required for the temperature difference $\Delta T=T_{w}-T_{S H Z}$ to drop to 10 , i.e., $10 \%$ of its initial value. The lag-factor, $j$ - value, on the other hand, indicates the duration of the lag-period. It is desirable to have both these factors as small as possible for a rapid heating of the slowest heating zone.

Figures 13 and 14 show the effects of $A, S$ and $\alpha$ on the resulting $f$-values and $j$-values. The respective $\mathrm{R}^{2}$ values (Tables 4 (a) and (b)) close to 1 indicate almost perfect linearity of these regions. In Figure 13, the 
$f$-value for a "short" can $(A=0.5)$ decreases as the inclination angle, $\alpha$ increases indicating an overall increase in the heating rate of the $S H Z$ following the lag-period. However, the $j$-value signifying the lagperiod is rather small for the vertical orientation $\left(\alpha=0^{\circ}\right)$ and it increases rapidly with the non-vertical orientations. Thus, although the heating rate increases, the lag-period also increases rapidly; and the net effect of these two factors is likely to denote a reduction in the sterilization time, as seen in Figure 11 (a). Conversely, for $A=1.0$ and 1.5 , there is an overall drop in both $f$ and $j$-values with the increasing $\alpha$. Since the lag-period is also reduced in this case, there is an overall reduction in the sterilization time as one inclines the "taller" ( $A=1$ and 1.5) cans from $\alpha=0^{\circ}$ to $\alpha=90^{\circ}$. This is also consistent with the trends seen in Figure 11 (a).

For the case of the horizontal orientation shown in Figure 14, one can see the overall reduction (after an initial rise) in $f$-value i.e., an increase in the SHZ heating rate after the lag period, with an increase in $S$ from 0.5 to 2 . However, the $j$-value is considerably smaller for $S=0.5$ which increases rapidly with $S$. The net effect, as can be seen in Figure 11 (b), is that shorter lag-period raises the SHZ temperature to the threshold value faster for the case $S=0.5$ than that at the higher values because the short lag-period compensates for the sluggishness of the heating process.

Conventionally, $f$ - and $j$-values are calculated from $\left(T_{W}-T_{S H Z}\right)$ vs. time plots. In the present study, $\left(T_{w}\right.$ $\left.T_{S H Z}\right)$ is rendered dimensionless as $u=\frac{T_{W}-T_{S H Z}}{T_{W}-T_{o}}$ and time as $t^{*}=t u_{c} L$. Thus, the dimensionless $f$ and $j-$ values are essentially the same as their dimensional counter-parts. However, they are applicable for different operating conditions in accordance with the values of the dimensionless groups. As a further clarification, the $f$ - and $j$ - values are obtained by fitting these plots to the following equation: $\log u=-\frac{t}{f}+\log j$

\section{8 Effect of Rheology on heat penetration}


The preceding discussion on the effect of geometry on the heating characteristics of liquid canned food is based on a shear-thinning model food, CMC $0.85 \% \mathrm{w} / \mathrm{w}$ solution $(\operatorname{Pr}=79481)$. But the rheology of liquid foods is indeed very diverse and the value of Prandtl number also varies from fluid to fluid (Steffe, 1996). It is clear from the study of Moraga et al. (2011) on the heating of different liquids in cans that as one decreases the value of Prandtl number, the heating rate increases. Since a low Prandtl number may signify a low heat capacity or a low effective viscosity or a high thermal conductivity or all of these together, all these conditions imply a faster heat transfer or penetration. Thus, the effect of Prandtl number at a fixed value of Grashof number and can geometry is easy to understand. The following discussion briefly illustrates the effect of rheology by comparing the trends for a shear-thinning fluid (CMC, $n=0.57$ ), a Newtonian fluid $(n=1)$ and a shear-thickening fluid $(n=1.5)$ for a constant Prandtl number $(\operatorname{Pr}=79481)$.

The heat penetration plots, Figure 15(a), show the effect of the power-law index, $n$ on the heating characteristics of liquid foods in vertical cans of three aspects ratios. As expected, the heat penetration rate is the fastest in a shear-thinning fluid while, it is the slowest for the shear-thickening fluid, as convection is suppressed by the increased viscosity in this case. This difference manifests via the differences in the lag time as it intuitively appears that convective motion will take longer to set in for a shear-thickening fluid. The effect of aspect ratio $A$ for vertical cylindrical cans is shown for the three hypothetical fluids, Figure 15(b). In line with the earlier discussion, the heat penetration rate correlates inversely with the value of $A$, i.e., it is faster in shorter cans. Figure 15(b) shows that the difference between the extremes $A=0.5$ and $A$ $=1.5$ for $n=0.57$ is smaller as compared to that in the case $n=1.5$. Similarly, in figure $15(\mathrm{c})$, the enhancement in the heat penetration rate by inclining a cylindrical can of $A=1.5$ from vertical to horizontal is slightly higher for $n=1.5$ than that for $n=0.57$, while, $n=1$ is an intermediate case.

Thus, the preceding discussion shows that the effects of geometry and orientation of the can is slightly stronger for a shear-thickening fluid than that for a shear-thinning one. Further comparisons show that the reduction in sterilization time due to the change in the value of $A$ from 1.5 to 0.5 for a vertical cylindrical can is $13.7 \%, 16.1 \%$ and $16.8 \%$ for $n=0.57, n=1$ and $n=1.5$ respectively. Similarly, changing the 
orientation of a cylindrical can of $A=1.5$ from vertical to horizontal reduces the sterilization time by $10.1 \%$, $11 \%$ and $11 \%$ respectively. Thus, it is fair to conclude that the effect of geometry and orientation of the can on the heating rate of the liquid food is significant and their severity depends on the value of the power-law index $n$ of the food.

Before leaving this section, it needs to be recognized here that the present study is mainly concerned with the heating of a non-Newtonian liquid food in cans of different geometries. In most practical situations, the actual shape of a can is influenced by many other considerations including ease of manufacturing and of use, aesthetics, cost of material and fabrication, etc. rather than by heat transfer considerations alone. It is also worthwhile to revisit some of the key assumptions inherent in this study. While the temperaturedependence of the viscosity has been incorporated in this work, limited simulations have also been carried out by varying the values of $C_{p}$ and $k$ by $\pm 5 \%$ around their mean values. These results were found to be scattered within $2-3 \%$ of the results obtained using the mean values of $C_{p}$ and $k$. Thus, this effect is expected to be small, though it may vary from one product to another depending upon the severity of their $C_{p}-T$ and $k-T$ relationship. Similarly, it is possible that there might be vapour space present in the can above the product. While such a vapour space might contribute to the overall thermal resistance, available limited experimental results (Mohamed, 2007) indicate that the average heat transfer coefficient varied only by $3 \%$ with vapour spaces of $10-20 \%$ of the height of the can and hence it appears to be a minor effect only. In any case, should this vary from one application to another, the present results thus provide the lower bound on the heating time, etc. Similarly, the choice of the initial temperature of $T_{o}=40^{\circ} \mathrm{C}$ may also seem arbitrary. Admittedly, this value may vary from one product to another, the present results are reported in terms of the dimensionless temperature and therefore are valid for other values of $T_{o}$ provided the other assumptions remain valid.

\section{CONCLUSIONS}

Geometry and orientation of the food cans have significant effect on the heating/penetration characteristics with respect to the thermal food treatment applications for a fluid of fixed properties. The heating process 
develops through an initial conduction-dominated phase paving the way for the onset of convection. The strength of convection current weakens with the decreasing temperature driving force as the liquid is gradually heated up. Eventually, it ceases completely when the temperature is uniform everywhere in the can. The flow patterns during the convection dominated phase, velocity vectors and velocity magnitudes contours, are strongly influenced by the can geometry. A low aspect ratio, $A=0.5$ ("short" can) allows the convective currents to form re-circulatory patterns with much ease as opposed to a high aspect ratio e.g., $A$ $=2$ ("very tall" can), for which the narrow shape allows strong currents only in the vertical direction. However, for a tall can in the horizontal orientation, due to the shorter vertical dimension and curved walls, the fluid travels in "circular" path thereby enhancing the strength of convection vis á vis better distribution of heat. The location, shape and size of the SHZ can be determined from the isotherm contours. The variation in the rate of evolution of SHZ temperature with time is due to the variation of the both heating rate $(f$-value) and lag-period ( $j$-value). Thus, sterilization time also varies with the geometrical parameters significantly. For instance, in the vertical orientation, "very tall" cylindrical cans $(A=2, S=1)$ require the longest sterilization time. However, aligning it in the horizontal orientation reduces the sterilization time by about $16 \%$ with respect to that in the vertical orientation. The sterilization time is further reduced by $22 \%$ if the aspect ratio $S$ is changed from 1 to 0.5 (cylindrical to elliptical). Inclination of a short can $(A=$ $0.5)$ increases the sterilization time. If the horizontal orientation is fixed, decreasing the value of $S$ reduces the sterilization time in all cases. The effect of geometry and orientation of the can on the heating rate of the liquid food is significant irrespective of the shear-rate dependent behavior of the fluid. The effect may be enhanced for shear-thickening fluids, i.e., $n>1$. In conclusion, the present study explores the geometrical and rheological aspects of canned food sterilization to suggest different possibilities for process optimization. It is expected that the numerical predictions reported herein will trigger experimental activity in this field thereby substantiating or refuting such predictions.

\section{ACKNOWLEDGMENTS}


2

3

4

5

6

7

8

9

10

11

12

13

14

15

16

17

18

19

20

21

22

23

24

25

26

27

28

29

30

31

32

33

34

35

36

37

38

39

40

41

42

43

44

45

46

47

48

RPC gratefully acknowledges the financial support of Department of Science and Technology, Government of India, New Delhi through the award of a J C Bose fellowship to him. We are also grateful to the anonymous reviewers for their constructive criticism of this work.

\section{REFERENCES}

Al-Baali, A.G.A.-G., \& Farid, M.M. (2006). Thermal Sterilization of Food, in: Sterilization of food in retort pouches, Food Engineering Series. Springer, Boston, MA, 1-16. 
Anand Paul, D., Anishaparvin, A., \& Anandharamakrishnan, C. (2011). Computational fluid dynamics studies on pasteurisation of canned milk. International Journal of Dairy Technology, 64, 305-313.

Augusto, P.E.D., \& Cristianini, M. (2012). Computational fluid dynamics evaluation of liquid food thermal process in a brick shaped package. The Journal of Food Science and Technology, 32, 134-141.

Augusto, P.E.D., Pinheiro, T.F., \& Cristianini, M. (2010). Using Computational fluid dynamics (CFD) for the evaluation of beer pasteurization: effect of orientation of cans. The Journal of Food Science and Technology, 30, 980-986.

Barakat, H.Z., \& Clark, J.A. (1966). Analytical and experimental study of the transient laminar natural convection flows in partially filled liquid containers. International Heat Transfer Conference II, $152-162$.

Bigelow, W.D., Bohart, G.S., Richardson, A.C., \& Ball, C.O. (1920). Heat penetration in processing canned foods. Research Laboratory, National Canners Association, Washington, D.C.

Bird, R.B., Armstrong, R.C., \& Hassager, O. (1987). Dynamics of Polymeric Liquids, Volume 1: Fluid Mechanics, (2nd ed.). Wiley-Interscience, New York.

Blaisdell, J.L. (1963). Natural convection heating of liquids in unagitated food containers. Michigan State University.

Datta, A. K. (1985). Numerical modeling of natural convection and conduction heat transfer in canned foods with application to online process control. Ph.D. Dissertation. University of Florida, Gainesville.

Engelman, M.S., \& Sani, R.L. (1983). Finite-element simulation of an in-package pasteurization process. Numerical Heat Transfer, 6, 41-54.

Evans, L.B., Reid, R.C., \& Drake, E.M. (1968). Transient natural convection in a vertical cylinder. AIChE Journal, 14, 251-259.

Fagerson, I.S., \& Esselen, W.B. (1950). Heat transfer in commercial glass containers during thermal processing. Food Technology, 4, 411-415.

Ghani, A.G., Farid, M.M., Chen, X.D., \& Richards, P. (2001). A computational fluid dynamics study on the effect of sterilization temperatures on bacteria deactivation and vitamin destruction. Proceedings of the Institution of Mechanical Engineers, Part E: Journal of Process Mechanical Engineering, 215, 9-17.

Ghani, A.G., Farid, M.M., \& Chen, X.D. (2002). Numerical simulation of transient temperature and velocity profiles in a horizontal can during sterilization using computational fluid dynamics. Journal of Food Engineering, 51, 77-83.

Ghani, A.G., Farid, M.M., Chen, X.D., \& Richards, P. (2001). Thermal sterilization of canned food in a 3-D pouch using computational fluid dynamics. Journal of Food Engineering, 48, 147-156. 
Gillespy, T.G. (1951). Estimation of the sterilizing values of processes as applied to canned foods. I- Packs heating by conduction. Journal of the Science of Food and Agriculture, 2, 107-125.

Gillespy, T.G. (1953). Estimation of the sterilizing values of processes as applied to canned foods. II Packs heating by conduction: complex processing conditions and value of coming-up time of retort. Journal of the Science of Food and Agriculture, 4, 553-565.

Hayakawa, K., \& Ball, C.O. (1971). Theoretical formulas for temperatures in cans of solid food and for evaluating various heat processes. Journal of Food Science, 2, 306-310.

Hiddink, J. (1975). Natural convection heating of liquids, with reference to sterilization of canned food. PhD Thesis, Wageningen, Netherlands. (Agric. Res. Rep. No. 839, Cent. Agric. Publ. Doc, Wageningen, Netherlands.)

Hiddink, J., Schenk, J., \& Bruin, S. (1976). Natural convection heating of liquids in closed containers. Applied Scientific Research, 32, 217-237.

Holdsworth, S.D., \& Simpson, R. (2007). Thermal Processing of Packaged Foods, (2nded.). Springer, New York.

Jackson, J.M., \& Olson, F.C.W. (1940). Thermal processing of canned foods in tin containers. Food Research, 5, 409-421.

Jones, D.E.A. (1931). Heat penetration by convection. Food Technology, 1, 63-65.

Kannan, A., \& Neeharika, K. (2007). Estimation of energy consumption in thermal sterilization of canned liquid foods in still retorts. Engineering Application of Computational Fluid Mechanics, 1, 288303.

Kannan, A., \& Sandaka, P.C.G. (2008). Heat transfer analysis of canned food sterilization in a still retort. Journal of Food Engineering, 88, 213-228.

Kızıltaş, S., Erdoğdu, F., Koray Palazoğlu, T. (2010). Simulation of heat transfer for solid-liquid food mixtures in cans and model validation under pasteurization conditions. Journal of Food Engineering, 97, $449-456$.

Kumar, A., \& Bhattacharya, M. (1991). Transient temperature and velocity profiles in a canned nonNewtonian liquid food during sterilization in a still-cook retort. International Journal of Heat and Mass Transfer, 34, 1083-1096.

Kumar, A., Bhattacharya, M., \& Blaylock, J. (1990). Numerical simulation of natural convection heating of canned thick viscous liquid food products. Journal of Food Science, 55, 1403-1411.

Lespinard, A.R., Mascheroni, R.H. (2012). Influence of the geometry aspect of jars on the heat transfer and flow pattern during sterilization of liquid foods. Journal of Food Process Engineering, 35, 751-762. 
Marra, F., \& Romano, V. (2003). A mathematical model to study the influence of wireless temperature sensor during assessment of canned food sterilization. Journal of Food Engineering, 59, 245-252.

Mohamed, I.O. (2007) Determination of an effective heat transfer coefficients for can headspace during thermal sterilization process. Journal of Food Engineering, 79, 1166-1171.

Moraga, N., Torres, A., Guarda, A., \& Galotto, M.J. (2011). Non-Newtonian canned liquid food, unsteady fluid mechanics and heat transfer prediction for pasteurization and sterilization. Journal of Food Process Engineering, 34, 2000-2025.

Moraga, N.O., Silva, L.A., \& Ortega, A. (2009). Unsteady natural convection heating of a canned nonNewtonian liquid food. Proceedings of the ASME 2009 Heat Transfer Summer Conference, 863871.

Rabiey, L., Flick, D., Duquenoy, A. (2007). 3D simulations of heat transfer and liquid flow during sterilisation of large particles in a cylindrical vertical can. Journal of Food Engineering, 82, 409-417.

Rao, M.A., \& Anantheswaran, R.C. (1988). Convective heat transfer to fluid foods in cans. Advances in Food Research. Academic Press, 39-84.

Reichert, J.E., \& Bielig, H.J. (1973). Sterilisationsbedingungen zur Herstellung von Kartoffelkonserven. Confructa $18,149-163$.

Rinaldi, M., Chiavaro, E., \& Massini, R. (2012). Real-time estimation of slowest heating point temperature and residual cooking time by coupling multipoint temperature measurement and mathematical modelling: Application to meat cooking automation. Food control, 23(2), 412-418.

Rinaldi, M., Malavasi, M., Cordioli, M., \& Barbanti, D. (2018). Investigation of influence of container geometry and starch concentration on thermal treated in-package food models by means of Computational Fluid Dynamics (CFD). Food and Bioproducts Processing, 108, 1-11.

Schultz, O.T., \& Olson, F.C.W. (1938). Thermal processing of canned foods in tin containers. Food Research, 3, 647-651.

Simpson, R. (2009). Engineering Aspects of Thermal Food Processing. CRC Press.

Steffe, J.F. (1996). Rheological methods in food process engineering, (2nd ed.). Freeman Press, East Lansing, MI.

Tani, S. (1940). Natural convection in can-shaped space. Bulletin of the Japanese Society of Scientific Fisheries, 8, 76-78.

Thompson, G.E. (1919). Temperature-time Relations in canned foods during sterilization. Journal of Industrial and Engineering Chemistry, 2, 657-664. 
Thorne, S.N., \& Jowitt, R. (1972). Fluidized bed heating; the influence of the outside heat transfer coefficient on the sterilizing value of the process. In Proc. Int. Symp. on Heat and Mass Transfer Problem in Food Engineering, Wageningen, The Netherlands.

Tritton, D.J. (1977). Physical Fluid Dynamics. Van Nostrand Reinhold Inc., U.S., New York.

Varma, M.N., \& Kannan, A. (2005). Enhanced food sterilization through inclination of the container walls and geometry modifications. International Journal of Heat and Mass Transfer, 48, 3753-3762.

Varma, M.N., \& Kannan, A. (200)6. CFD studies on natural convective heating of canned food in conical and cylindrical containers. Journal of Food Engineering, 77, 1024-1036.

Zechman, L.G., \& Pflug, I.J. (1989). Location of the slowest heating zone for natural-convection heating fluids in metal containers. Journal of Food Science, 54, 205-209.

Table 1. Thermo-physical properties of CMC $0.85 \% \mathrm{w} / \mathrm{w}$ solution.

$$
\begin{array}{ccc}
K_{o} & \left(\mathrm{~Pa} . \mathrm{s}^{\mathrm{n}}\right) & \mathbf{0 . 0 0 2 2 3 2} \\
E_{a} & (\mathrm{~J} / \mathrm{g} \mathrm{mol}) & 17521.8
\end{array}
$$




$\begin{array}{ccc}R & (\mathrm{~J} / \mathrm{g} \text { mol. K }) & 8.314 \\ n & (\text { dimensionless }) & 0.57 \\ C_{p} & \left(\mathrm{~J} / \mathrm{kg}^{\circ} \mathrm{C}\right) & 4100 \\ k & \left(\mathrm{~W} / \mathrm{m}^{\circ} \mathrm{C}\right) & 0.7 \\ \rho_{o} & \left(\mathrm{~kg} / \mathrm{m}^{3}\right) & 950 \\ \beta & \left({ }^{\circ} \mathrm{C}^{-1}\right) & 0.0002 \\ \mu_{o} & (\mathrm{~Pa} . \mathrm{s}) & 13.58\end{array}$

Table 2. Values of fitted constants in eq. (15)

\begin{tabular}{cccc}
\hline \multirow{2}{*}{ Constants } & \multicolumn{3}{c}{$N u_{\text {avg }}$} \\
\cline { 2 - 4 } & $\alpha=0^{0}$ & $\alpha=45^{0}$ & $\alpha=90^{0}$ \\
\hline$a$ & 41.990 & 43.110 & 43.230 \\
$b$ & $3.26 \mathrm{E}-02$ & $3.47 \mathrm{E}-02$ & $3.62 \mathrm{E}-02$ \\
$c$ & 9.3857 & 9.8558 & 10.3502 \\
$d$ & $1.69 \mathrm{E}-07$ & $2.05 \mathrm{E}-07$ & $2.25 \mathrm{E}-07$ \\
$e$ & 8.5347 & 8.6943 & 8.6405 \\
\hline$\delta_{\text {avg }}$ & $2.57 \%$ & $1.87 \%$ & $1.88 \%$ \\
\hline$\delta_{\max }$ & $5.36 \%$ & $7.40 \%$ & $5.53 \%$ \\
\hline Data point & 75 & 75 & 75 \\
\hline
\end{tabular}

Table 3 (a). Effect of geometry and orientation on sterilization time (dimensionless) at $S=1$.

$\begin{array}{cccc}\alpha & A=0.5 & A=1 & A=1.5 \\ 0^{\circ} & 1135.30 & 1283.72 & 1314.63 \\ 15^{\circ} & 1232.04 & 1313.64 & 1325.61 \\ 30^{\circ} & 1280.12 & 1328.38 & 1324.48 \\ 45^{\circ} & 1267.43 & 1322.41 & 1323.62\end{array}$




$\begin{array}{llll}60^{\circ} & 1263.48 & 1304.75 & 1296.02 \\ 75^{\circ} & 1245.82 & 1282.25 & 1257.65 \\ 90^{\circ} & 1234.88 & 1234.75 & 1165.65\end{array}$

Table 3 (b). Effect of geometry and orientation on sterilization time (dimensionless) at $\alpha=90^{\circ}$.

$\begin{array}{cccc}S & A=0.5 & A=1 & A=2 \\ 0.5 & 1118.13 & 1130.86 & 1025.95 \\ 0.75 & 1194.92 & 1199.77 & 1076.08 \\ 1 & 1235.16 & 1234.35 & 1097.84 \\ 1.25 & 1255.60 & 1250.86 & 1118.08 \\ 1.5 & 1269.08 & 1258.38 & 1119.14 \\ 1.75 & 1275.29 & 1258.23 & 1108.29 \\ 2 & 1275.14 & 1249.09 & 1096.12\end{array}$

Table 3 (c). Effect of geometry and orientation on sterilization time (dimensionless) at $S=1$.

$\begin{array}{ccc}A & \alpha=0^{\circ} & \alpha=90^{\circ} \\ 0.5 & 1135.12 & 1232.96 \\ 0.75 & 1241.17 & 1251.77 \\ 1 & 1282.79 & 1232.91 \\ 1.25 & 1300.09 & 1198.00 \\ 1.5 & 1314.05 & 1165.92 \\ 1.75 & 1308.11 & 1133.63 \\ 2 & 1305.01 & 1097.61\end{array}$

Table 4 (a). $\mathrm{R}^{2}$ values corresponding to Figure 13. 


\begin{tabular}{|c|c|c|c|c|c|c|}
\hline $\boldsymbol{\alpha}=\mathbf{0}^{\mathbf{0}}$ & $\boldsymbol{\alpha}=\mathbf{1 5}^{\mathbf{0}}$ & $\boldsymbol{\alpha}=\mathbf{3 0}^{\mathbf{0}}$ & $\boldsymbol{\alpha}=\mathbf{4 5}^{\mathbf{0}}$ & $\boldsymbol{\alpha}=\mathbf{6 0}^{\mathbf{0}}$ & $\boldsymbol{\alpha}=\mathbf{7 5}^{\mathbf{0}}$ & $\boldsymbol{\alpha}=\mathbf{9 0}^{\mathbf{0}}$ \\
\hline 0.9849 & 0.9895 & 0.9925 & 0.9931 & 0.993 & 0.9934 & 0.9929 \\
\hline 0.9872 & 0.9893 & 0.9902 & 0.9903 & 0.9896 & 0.9884 & 0.9859 \\
\hline 0.9921 & 0.993 & 0.993 & 0.9933 & 0.9925 & 0.9907 & 0.9858 \\
\hline
\end{tabular}

Table 4 (b). $\mathrm{R}^{2}$ values corresponding to Figure 14.

\begin{tabular}{|c|c|c|c|c|c|c|}
\hline $\boldsymbol{S}=\mathbf{0 . 5}$ & $\boldsymbol{S}=\mathbf{0 . 7 5}$ & $\boldsymbol{S}=\mathbf{1 . 0}$ & $\boldsymbol{S}=\mathbf{1 . 2 5}$ & $\boldsymbol{S}=\mathbf{1 . 5}$ & $\boldsymbol{S}=\mathbf{1 . 7 5}$ & $\boldsymbol{S}=\mathbf{2 . 0}$ \\
\hline 0.991 & 0.9927 & 0.993 & 0.9943 & 0.9953 & 0.9959 & 0.9962 \\
\hline 0.9842 & 0.9844 & 0.9861 & 0.9876 & 0.9892 & 0.9905 & 0.9905 \\
\hline 0.9913 & 0.988 & 0.988 & 0.9888 & 0.9898 & 0.9905 & 0.9917 \\
\hline
\end{tabular}




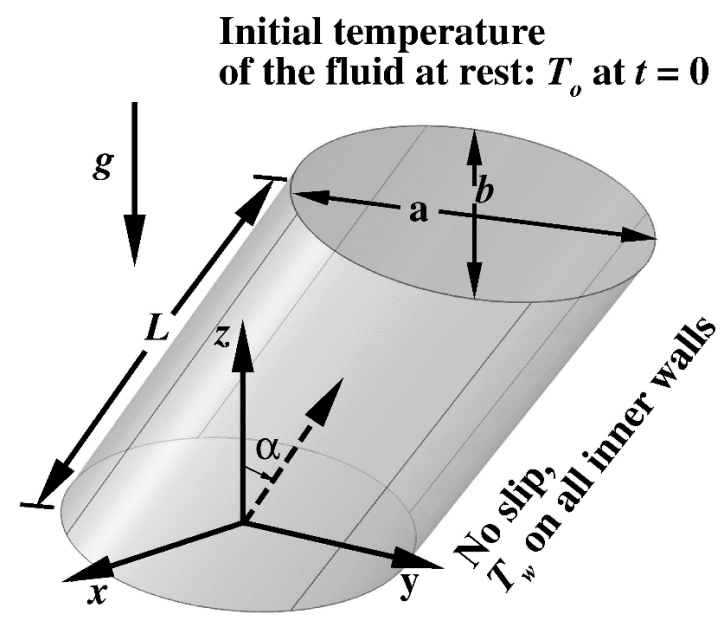

Figure 1. Food can schematic with boundary and initial conditions
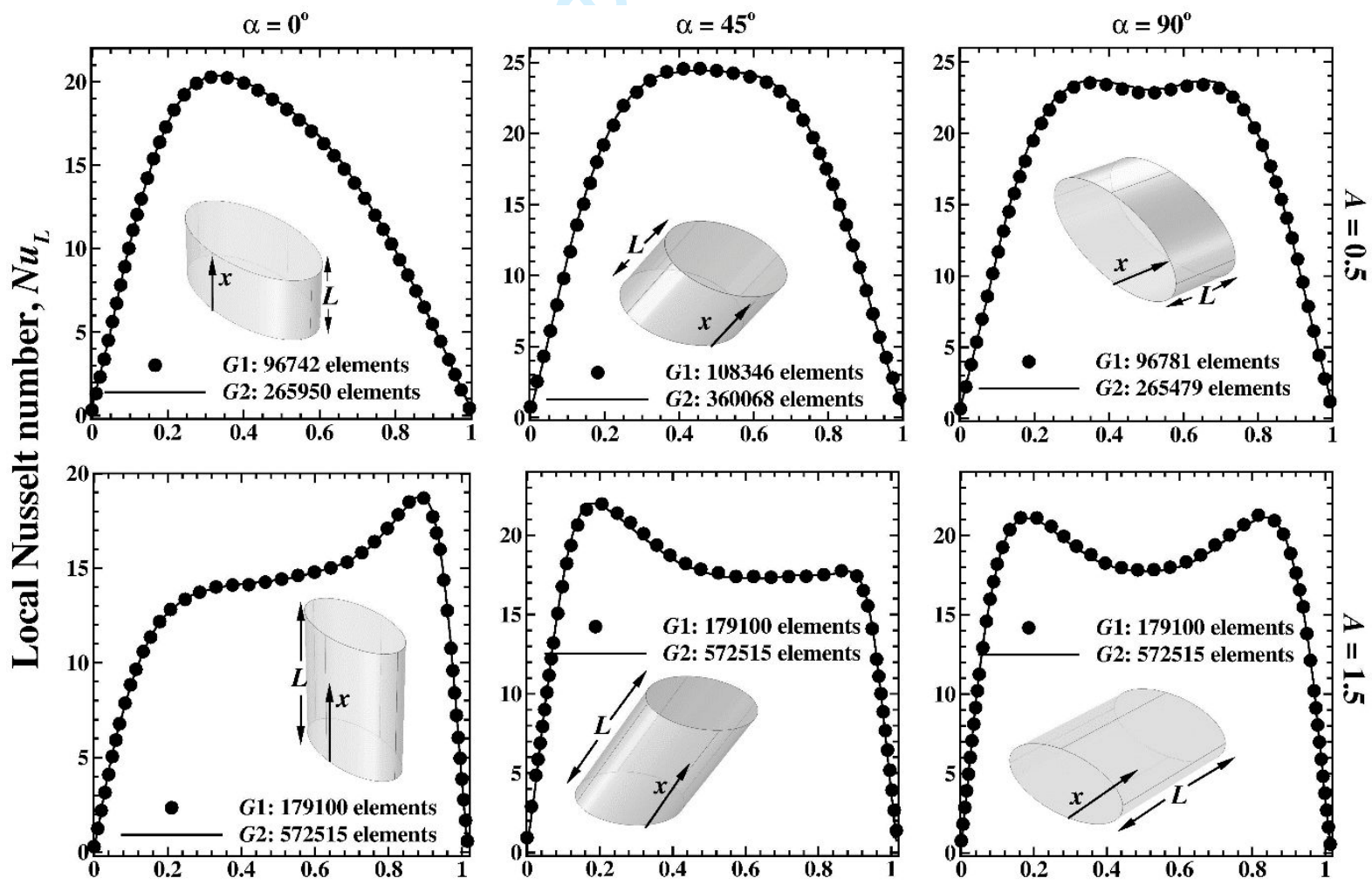

Distance along the wall (dimensionless), $x / L$

Figure 2. Grid independence test for CMC in elliptical cans: local Nusselt number at $t * 207.5$

(Time stepping scheme: Generalized alpha, $\Delta t=0.1 \mathrm{~s}$ ). 

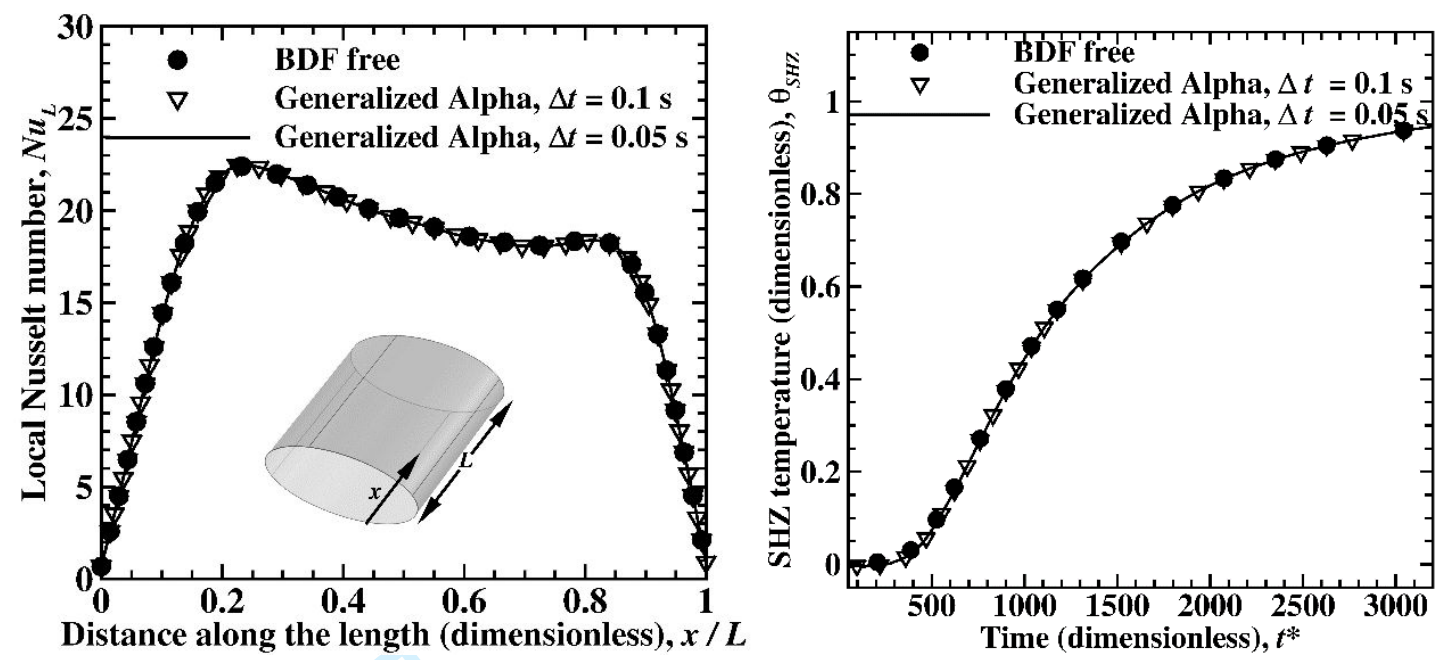

Figure 3. Time-step independence test for CMC in a can with $A=1, S=0.5, \alpha=45^{\circ}$.
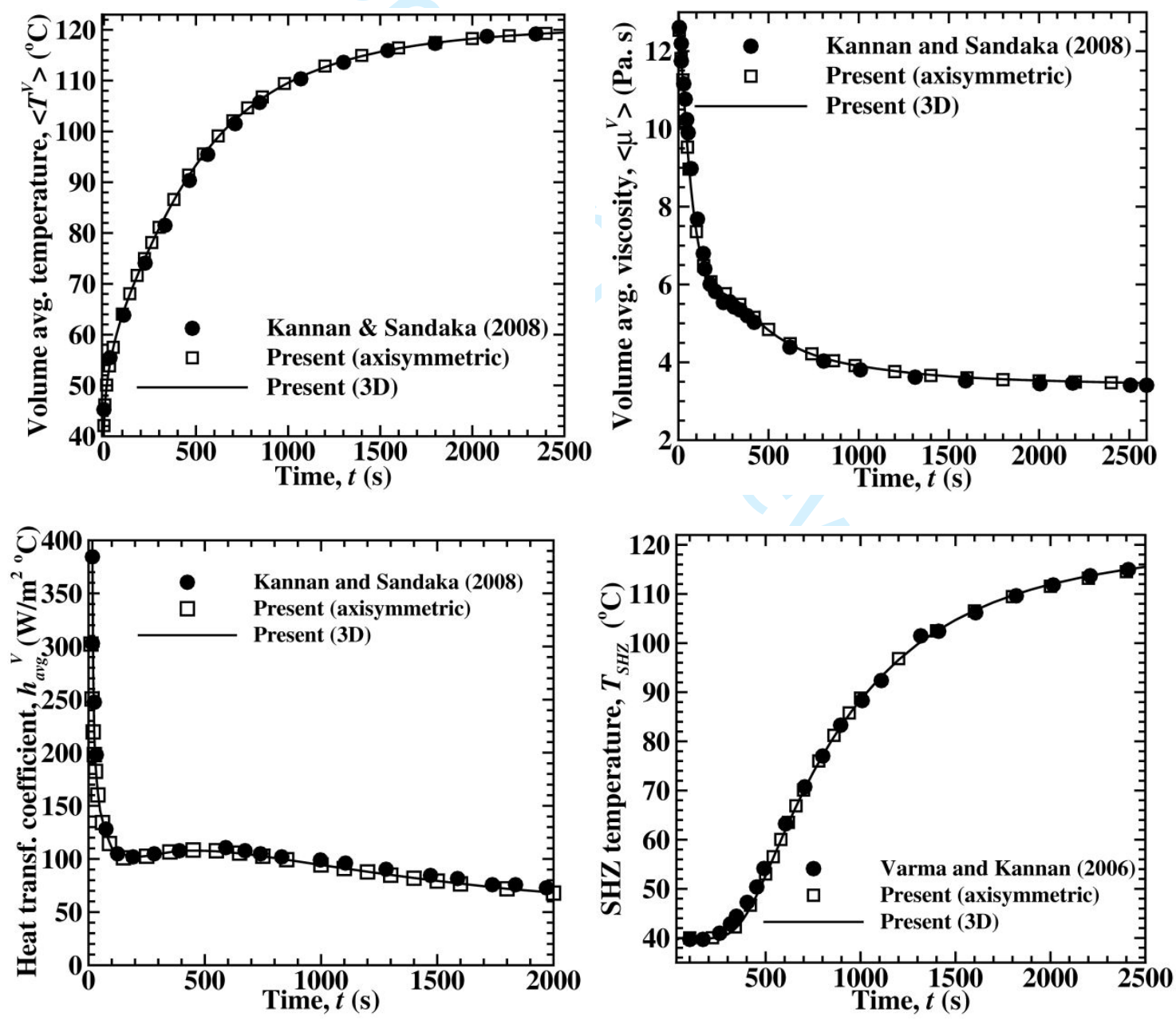

Figure 4. Vertical cylindrical can (Axisymmetric), Fluid: CMC $0.85 \% \mathrm{w} / \mathrm{w}$ solution. 
(a)
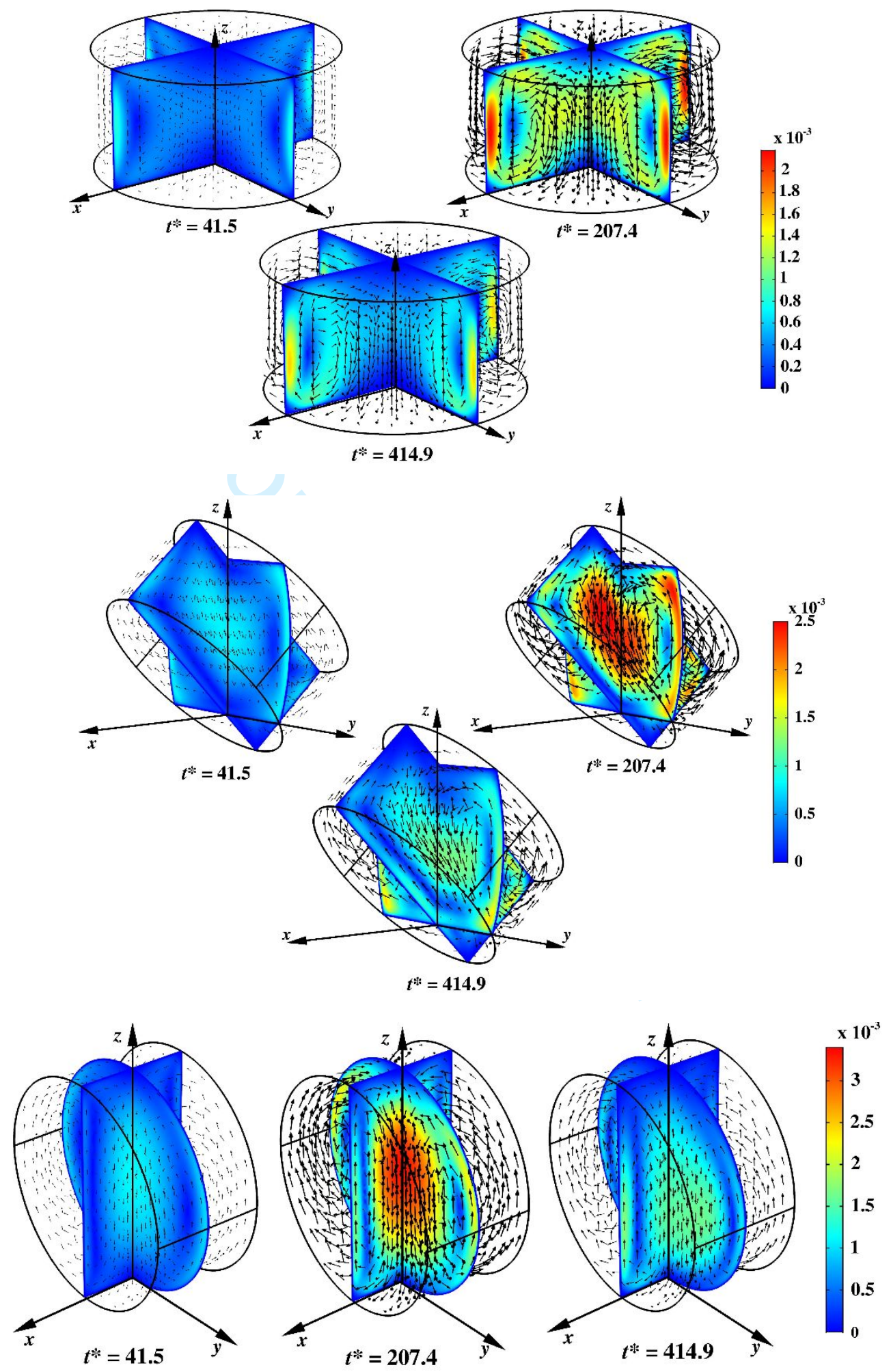
(b)
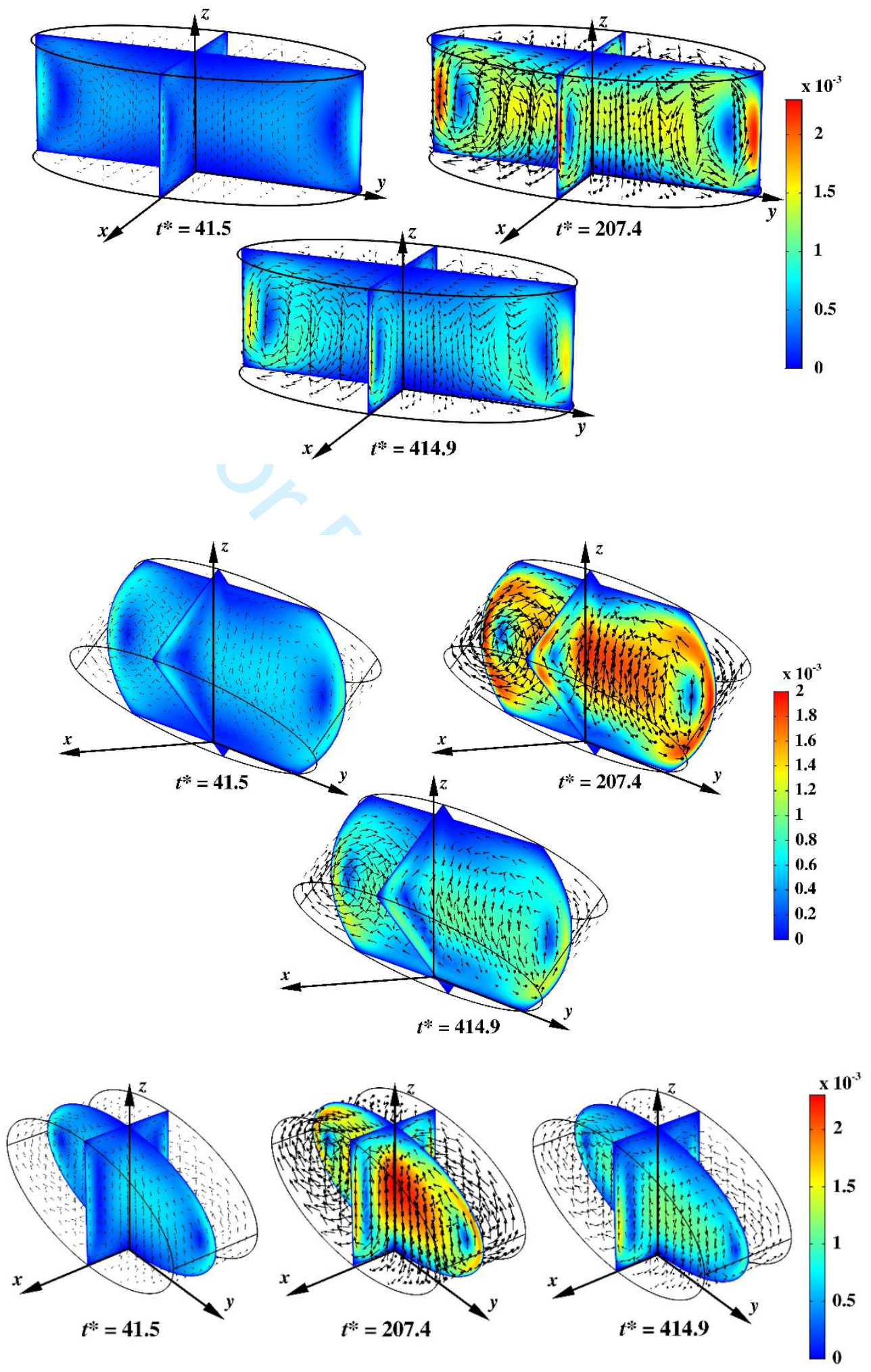

Figure 5. Velocity vectors and velocity magnitude contours (unit: $\mathrm{m} / \mathrm{s}$ ) for $A=0.5$ and $(a) S=1$ (b) $S=$ 0.5 . 
(a)
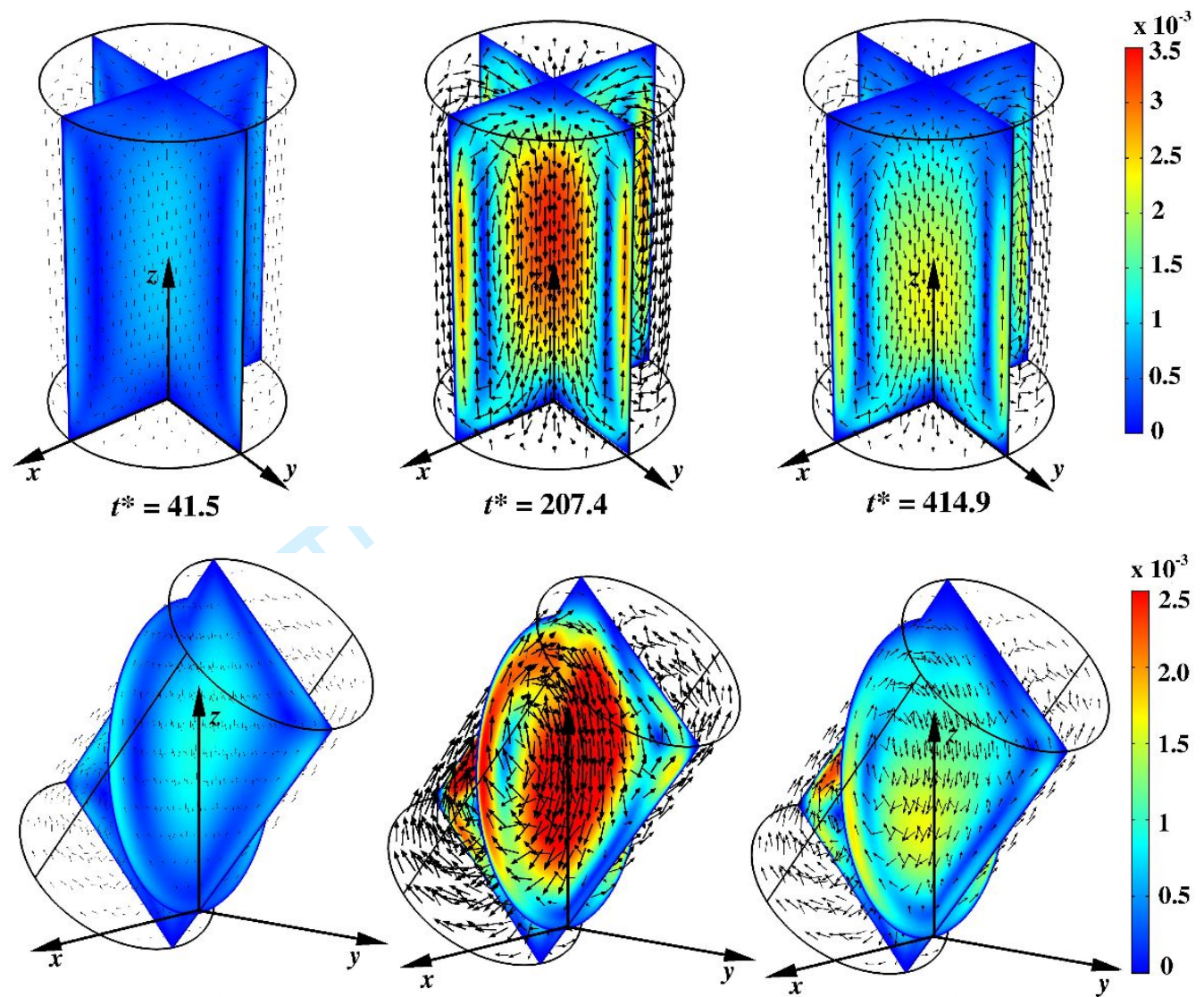

$t^{*}=41.5$
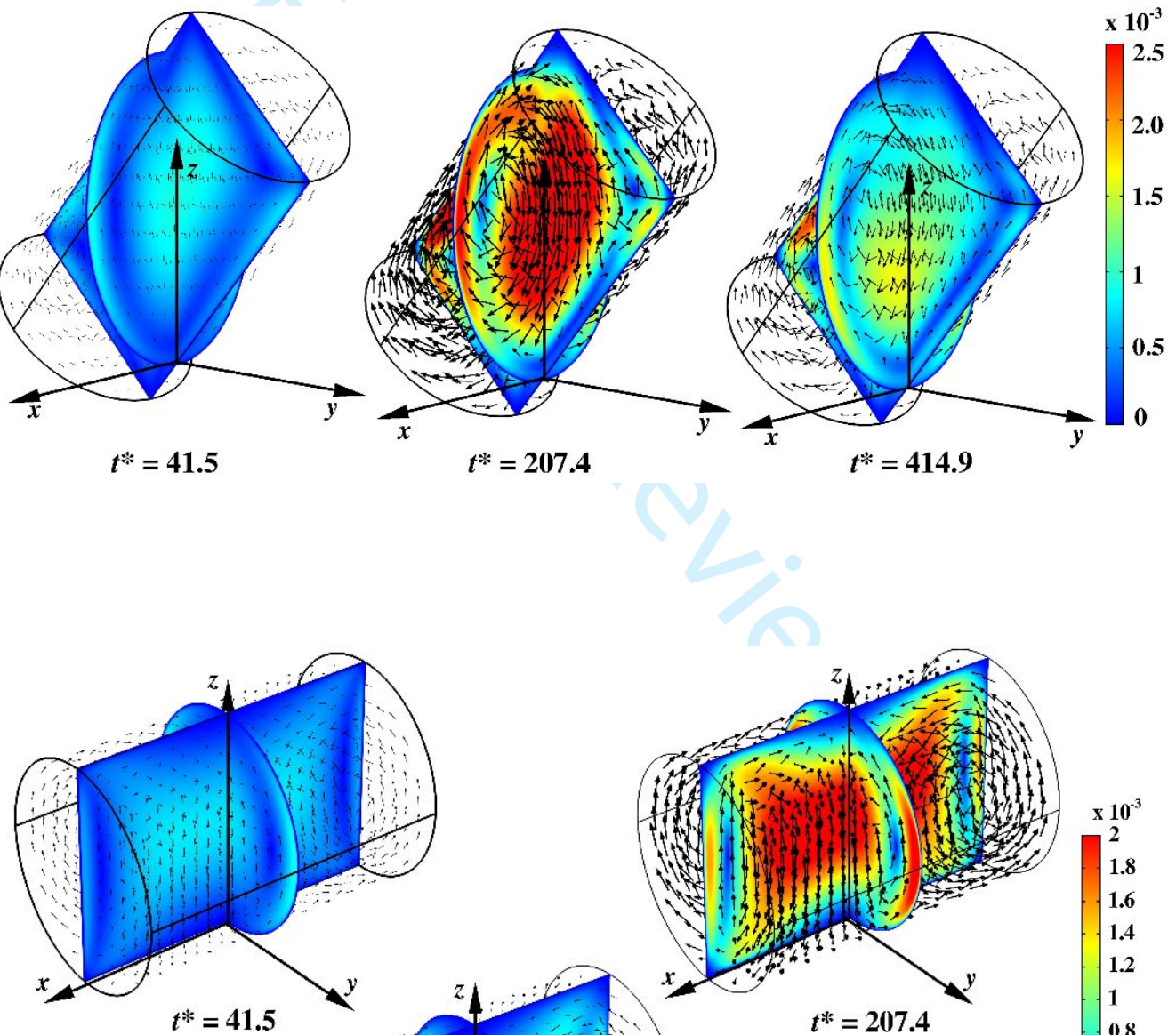

$t^{*}=41.5$
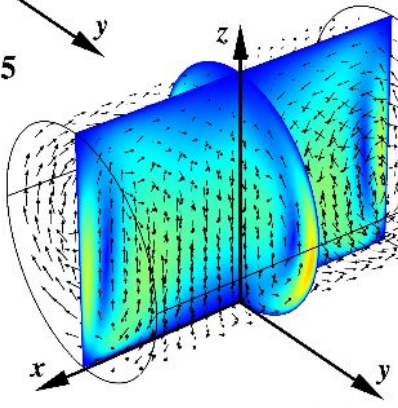

$t^{*}=414.9$

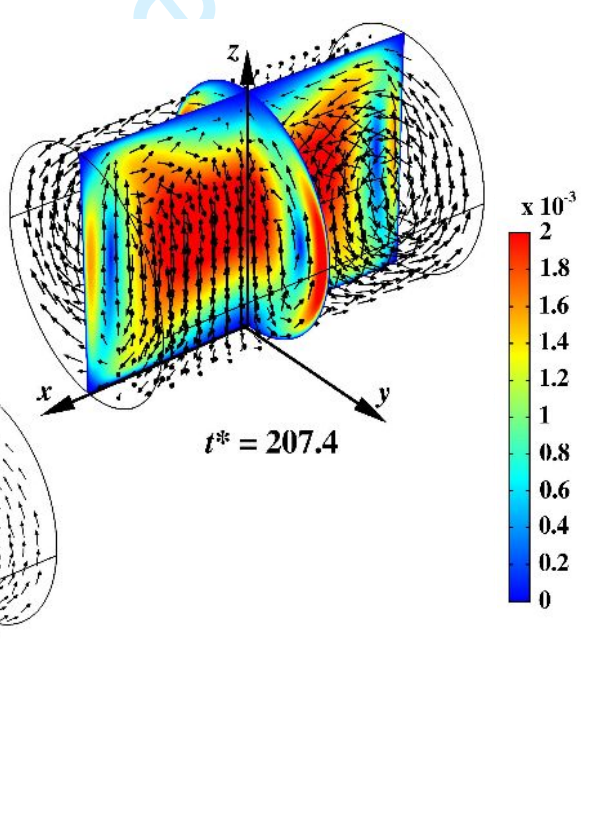


(b)

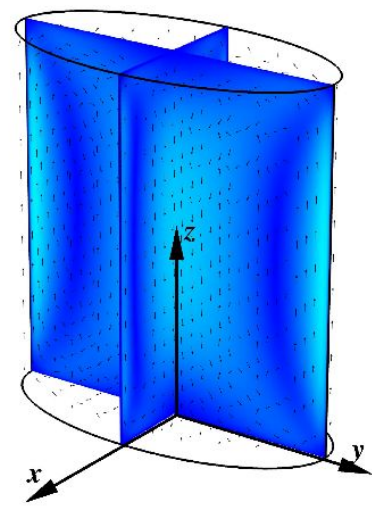

$t^{*}=41.5$

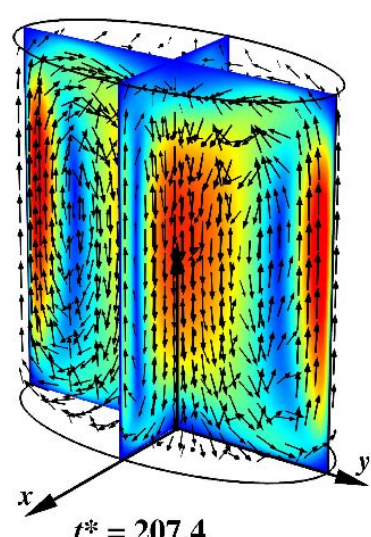

$t^{*}=\mathbf{2 0 7 . 4}$
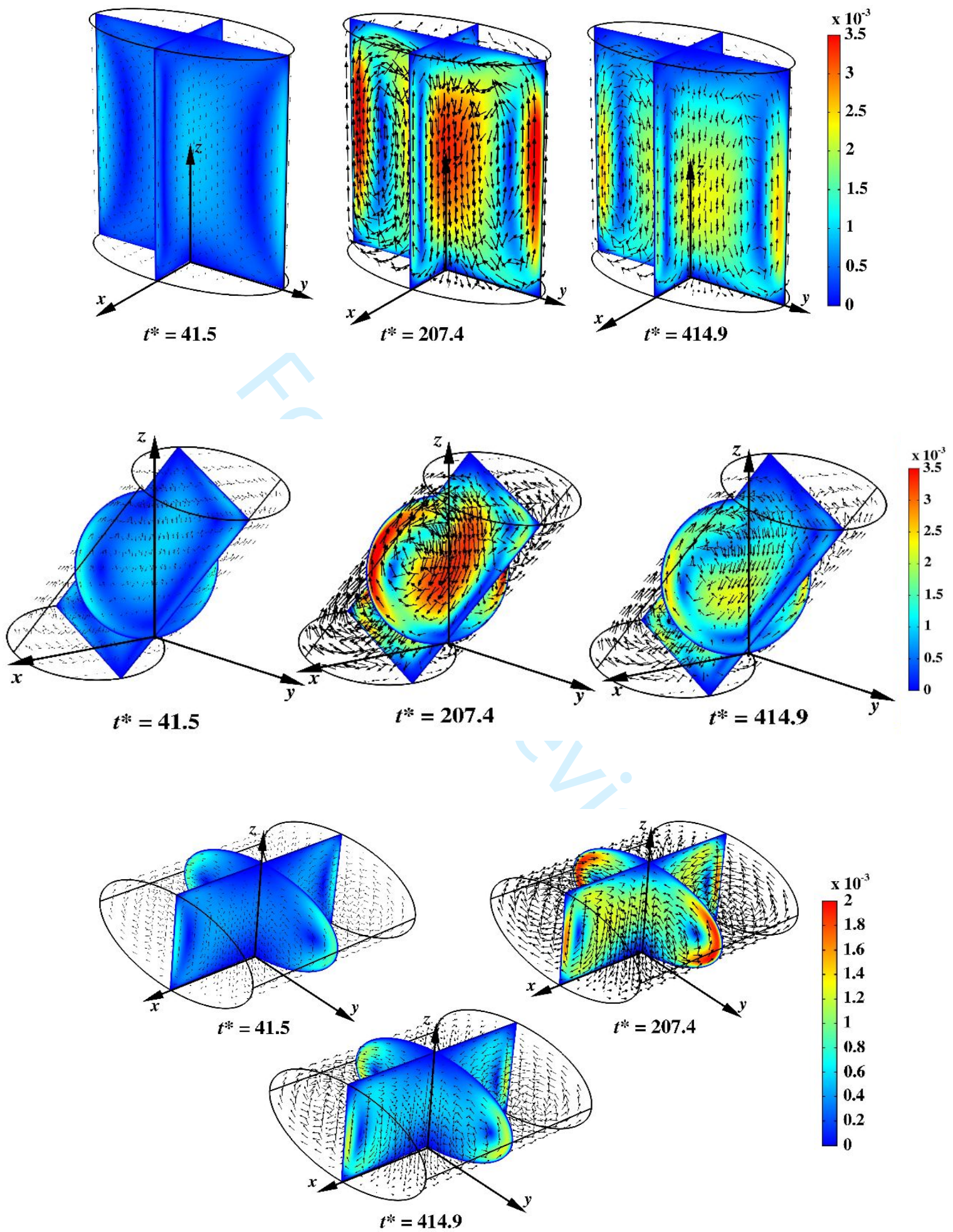

Figure 6. Velocity vectors and velocity magnitude (unit: $\mathrm{m} / \mathrm{s}$ ) contours for $A=1.5$ and $(a) S=1(b) S=$ 0.5 
(a)
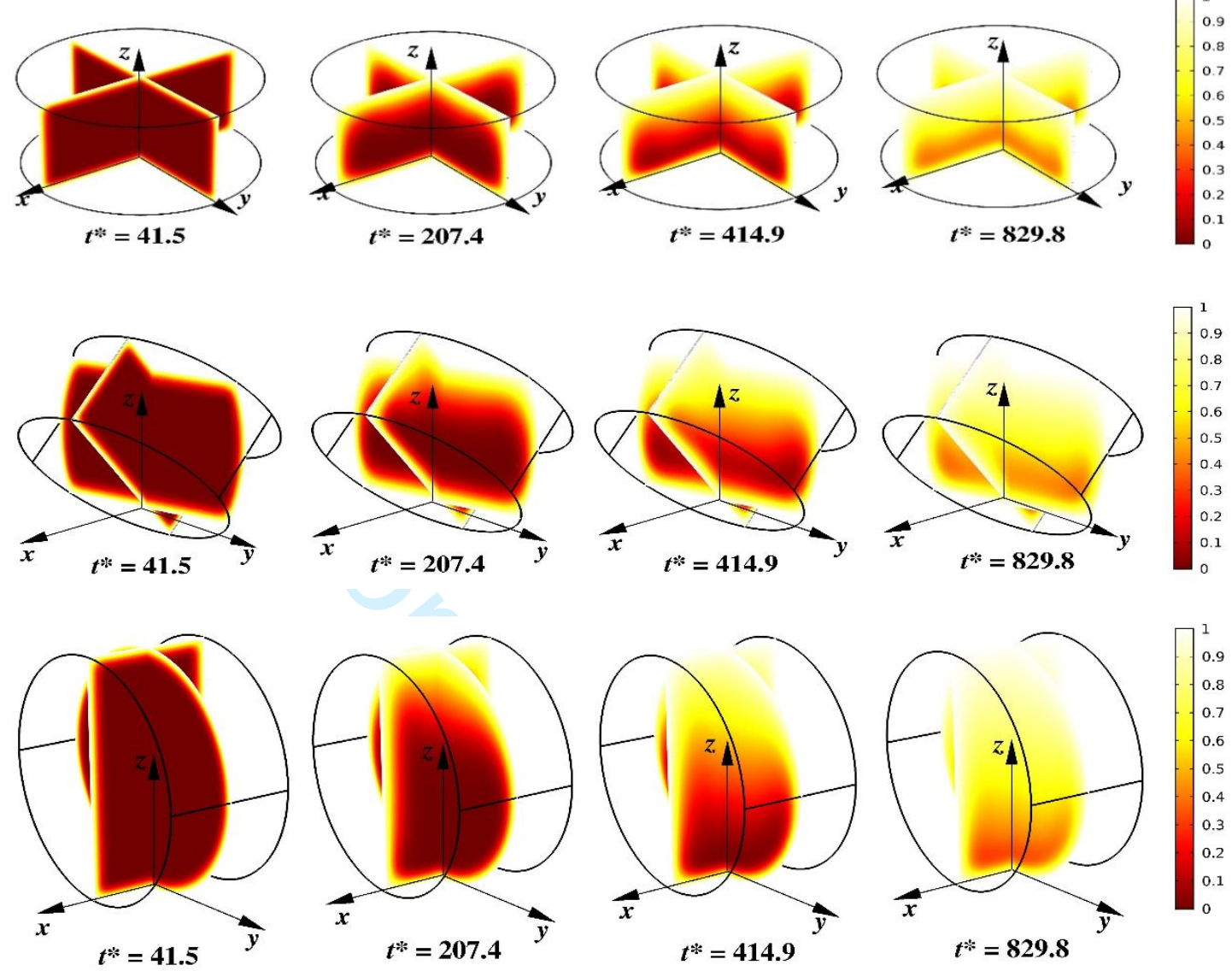

(b) 


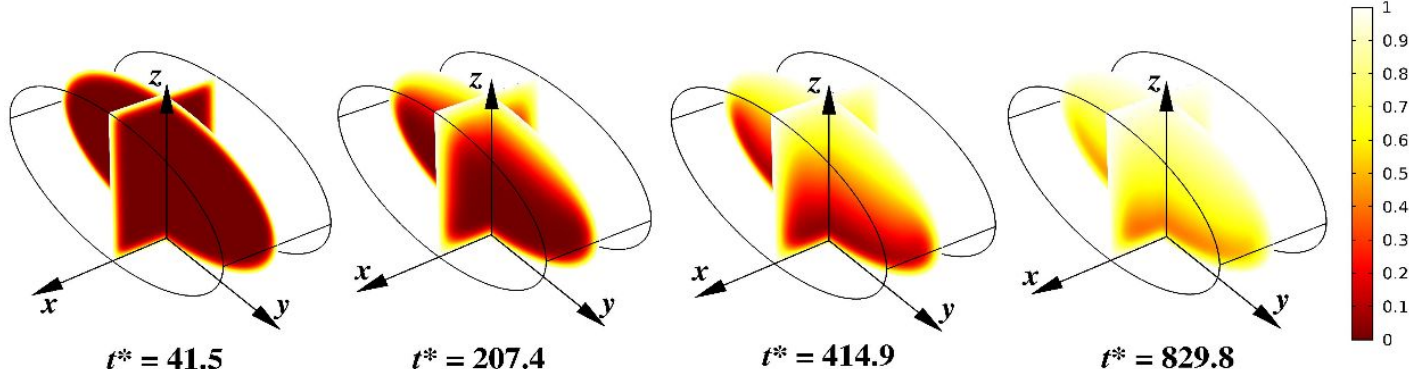

Figure 7. Isotherm contours (dimensionless) for $A=0.5$ and (a) $S=1(b) S=0.5$.

(a)
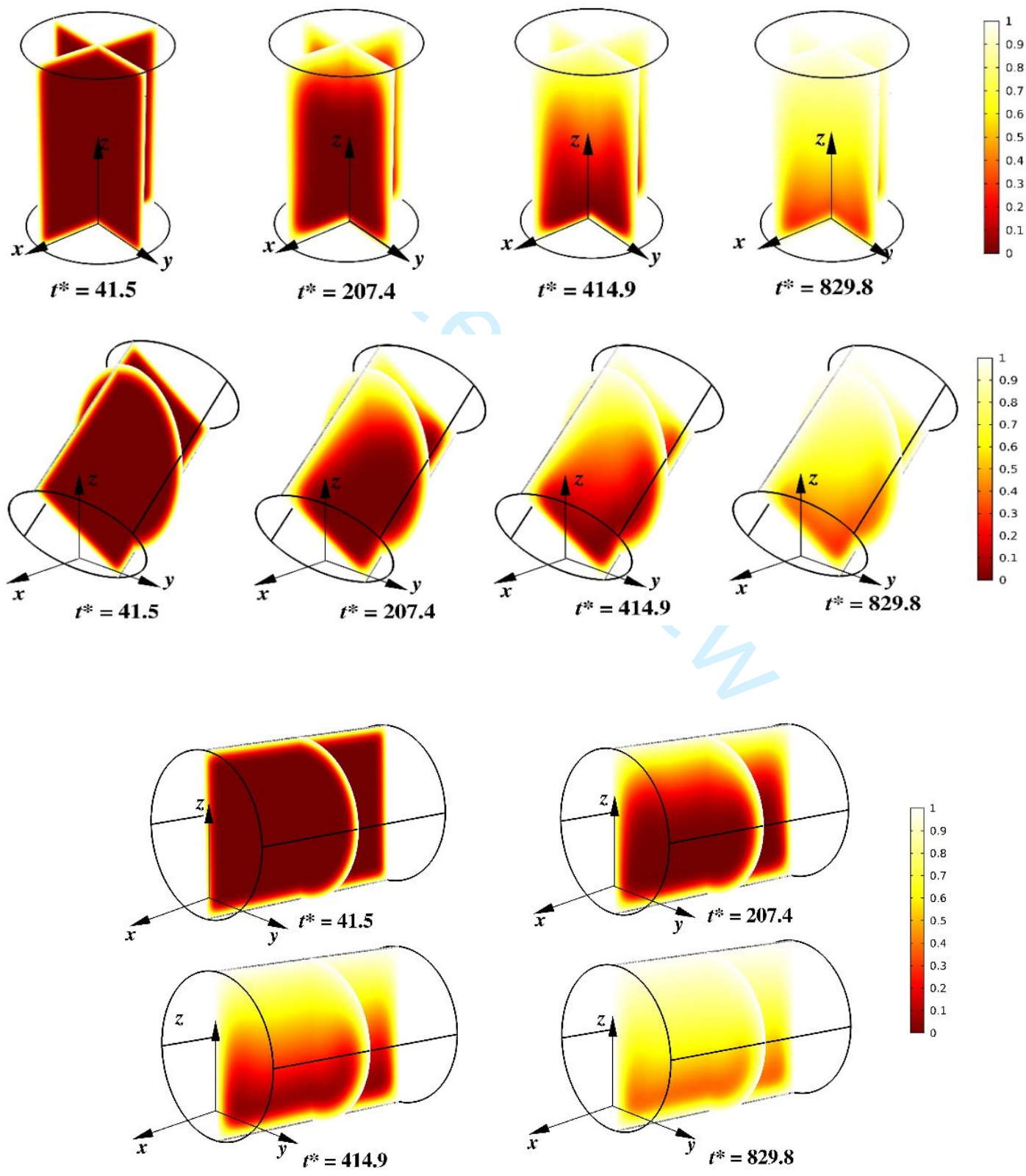
(b)
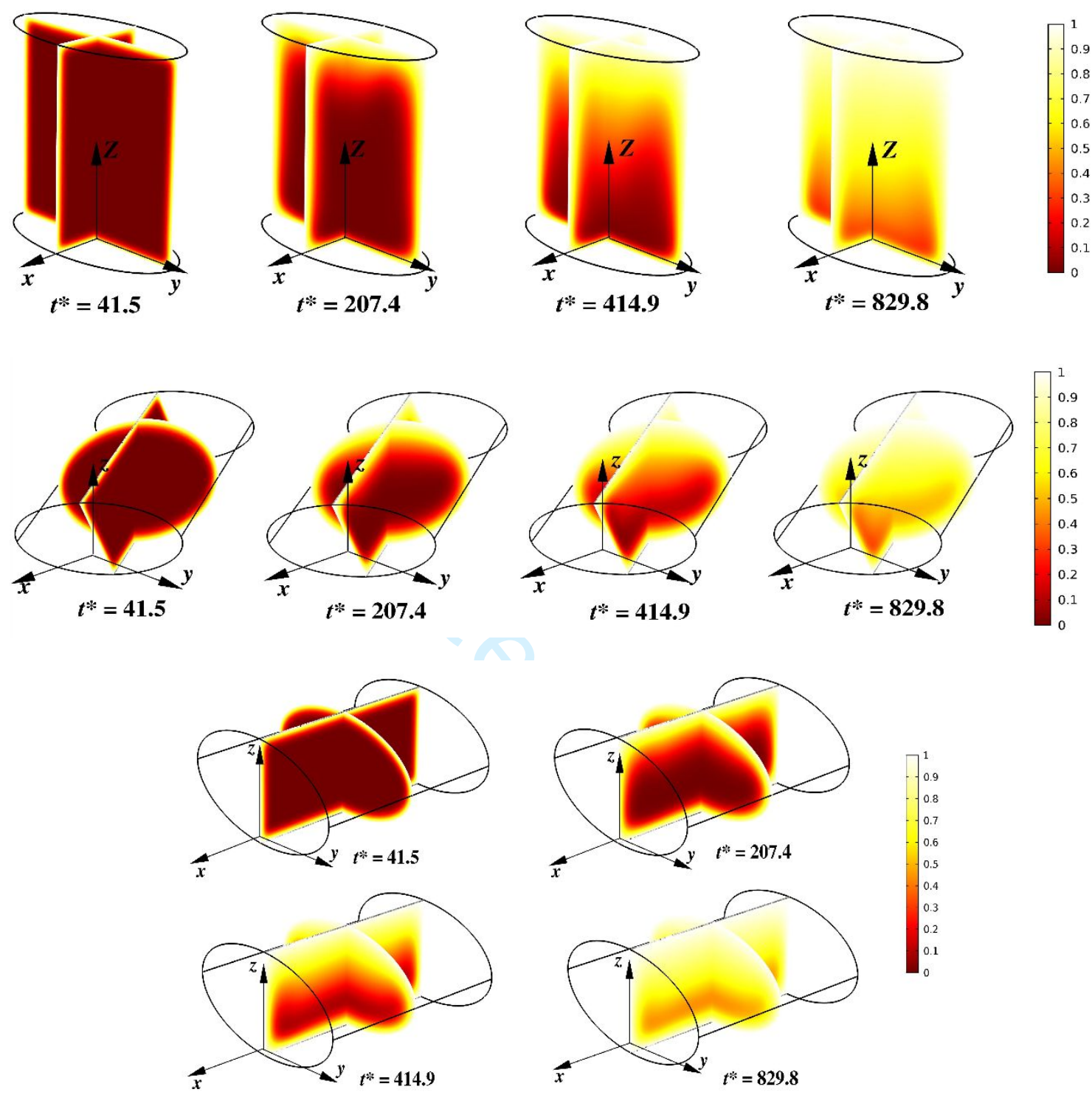

Figure 8. Isotherm contours (dimensionless) for $A=1.5$ and ( $a$ ) $S=1$ (b) $S=0.5$. 

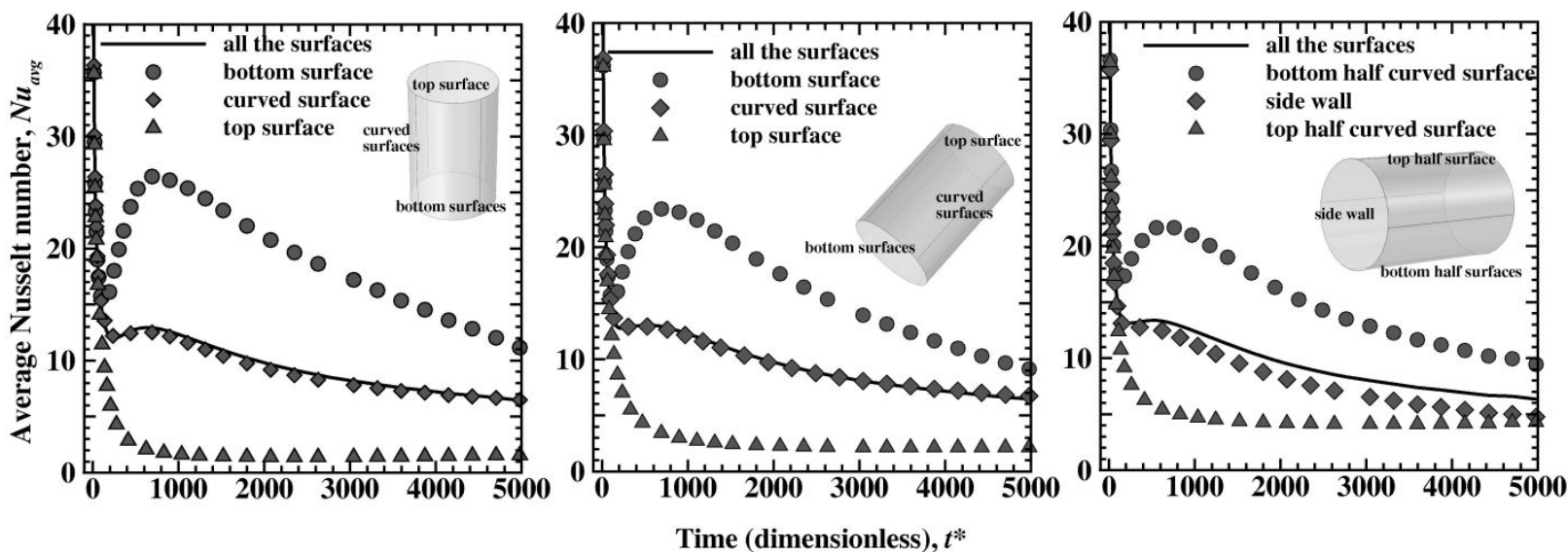

Figure 9. Vertical cylindrical can with $\mathrm{A}=1.5$., Fluid: $\mathrm{CMC} 0.85 \% \mathrm{w} / \mathrm{w}$ solution.
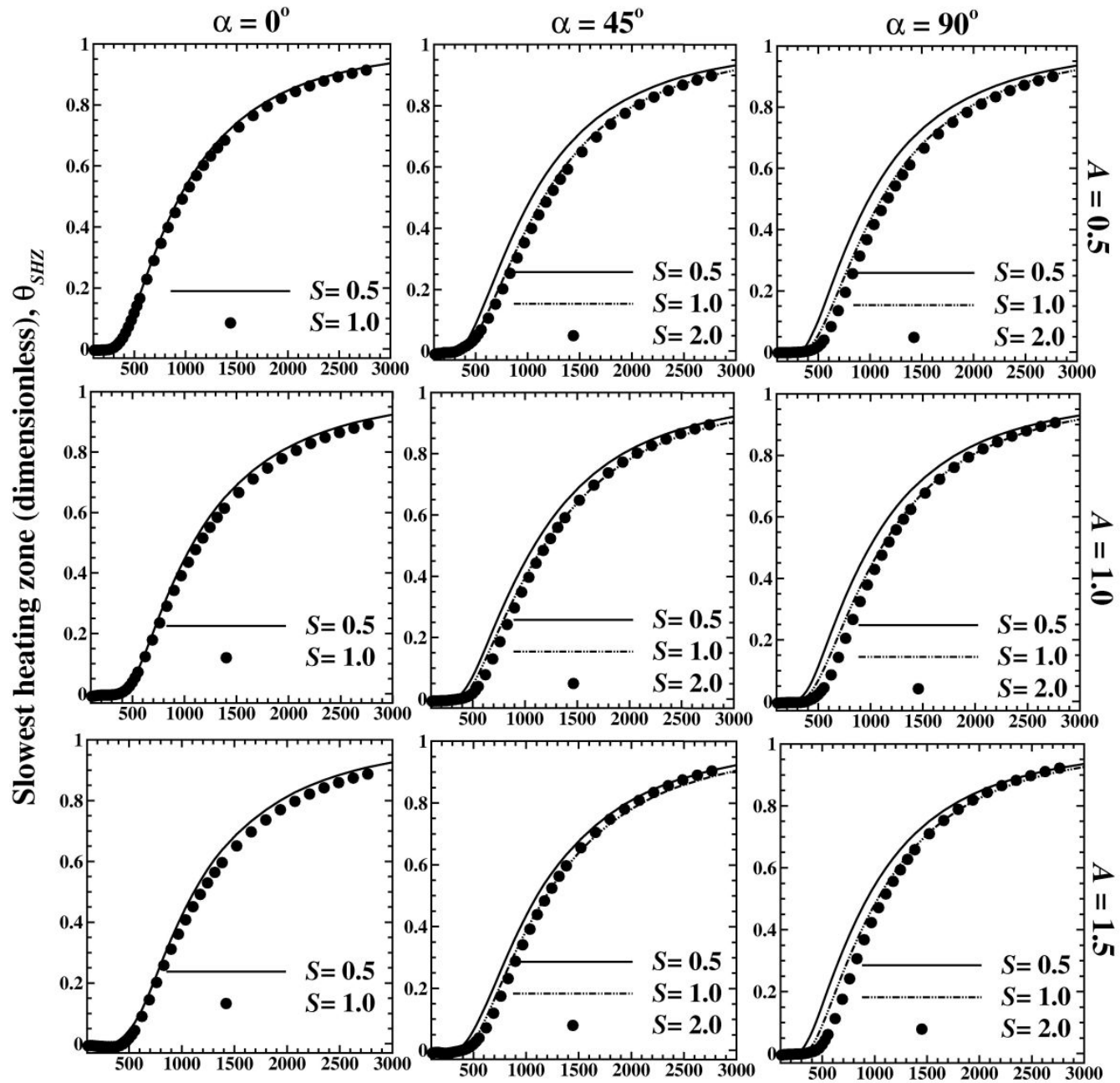

Time (dimensionless), $t$ *

Figure 10. Evolution of Slowest Heating Zone (SHZ) temperature with time for different cases. 
(a)

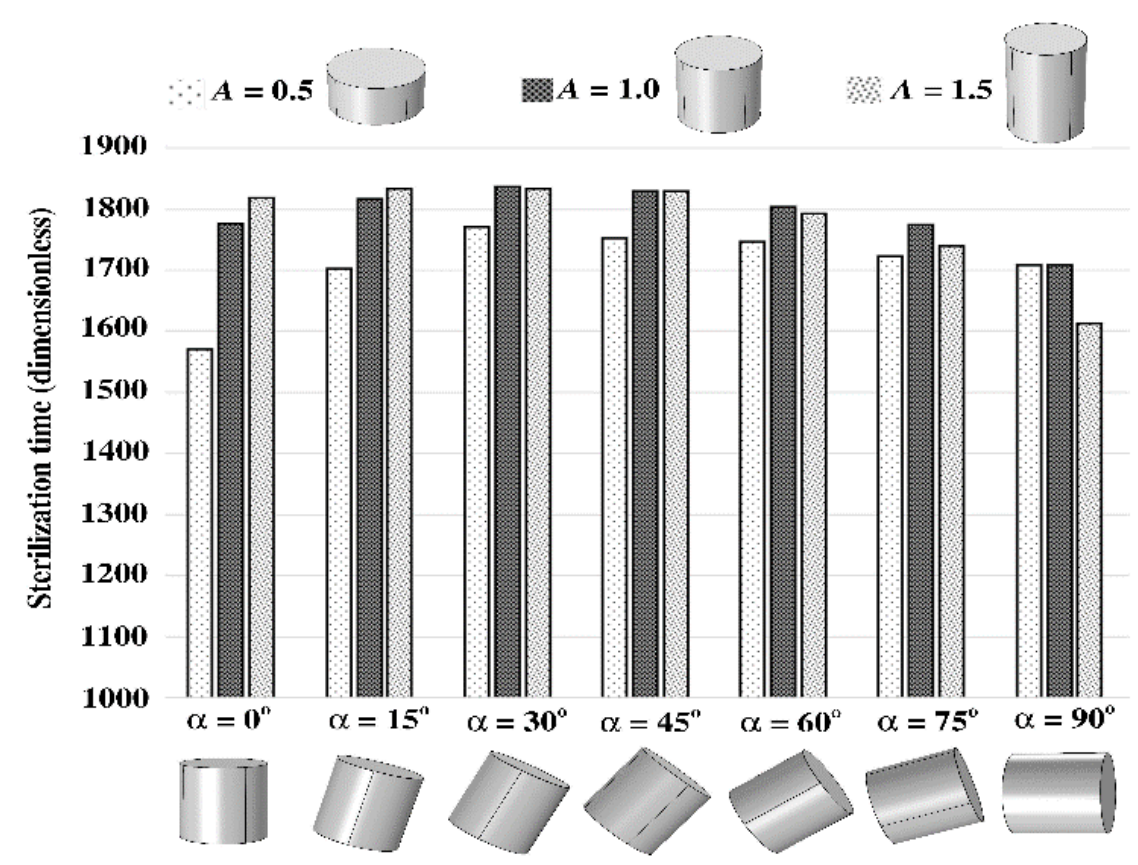

(b)

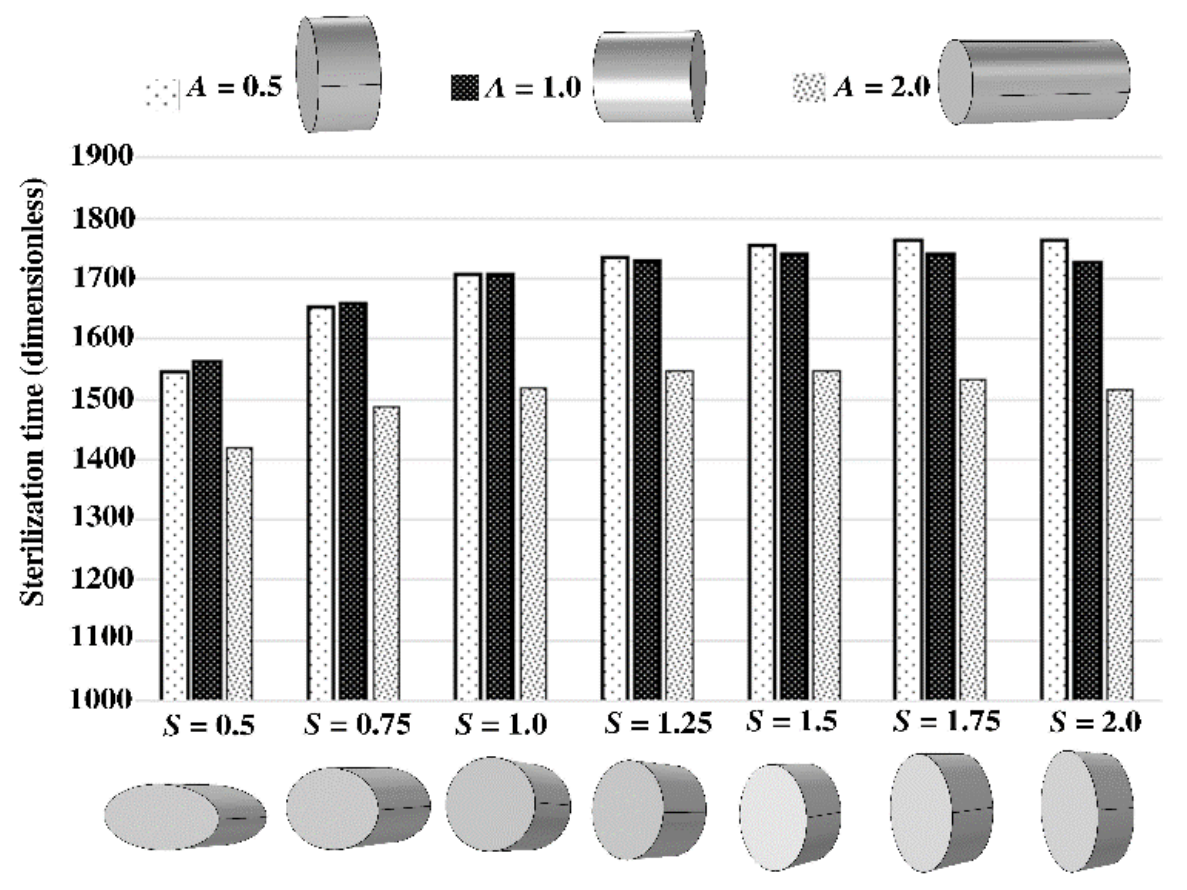


(c)

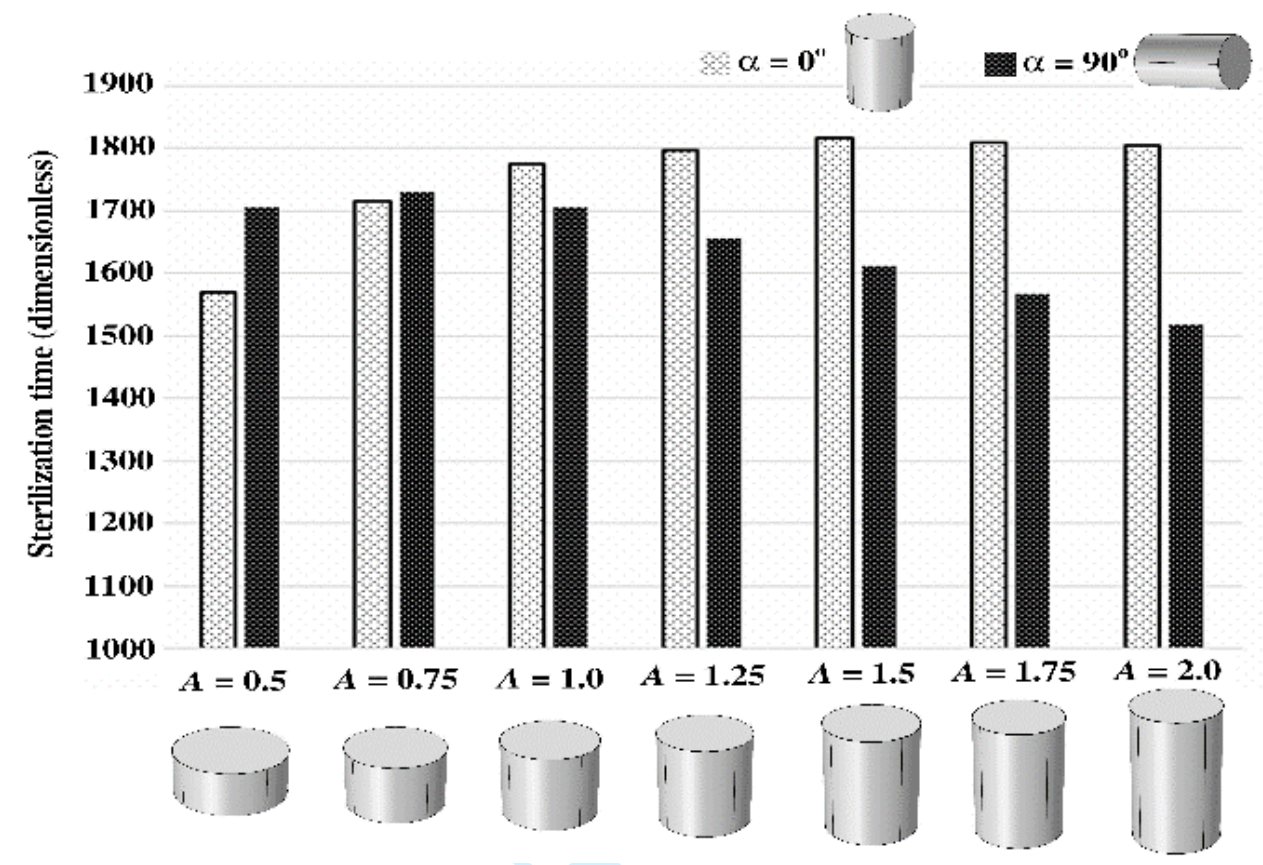

Figure 11. Effect of geometry and orientation on sterilization time 

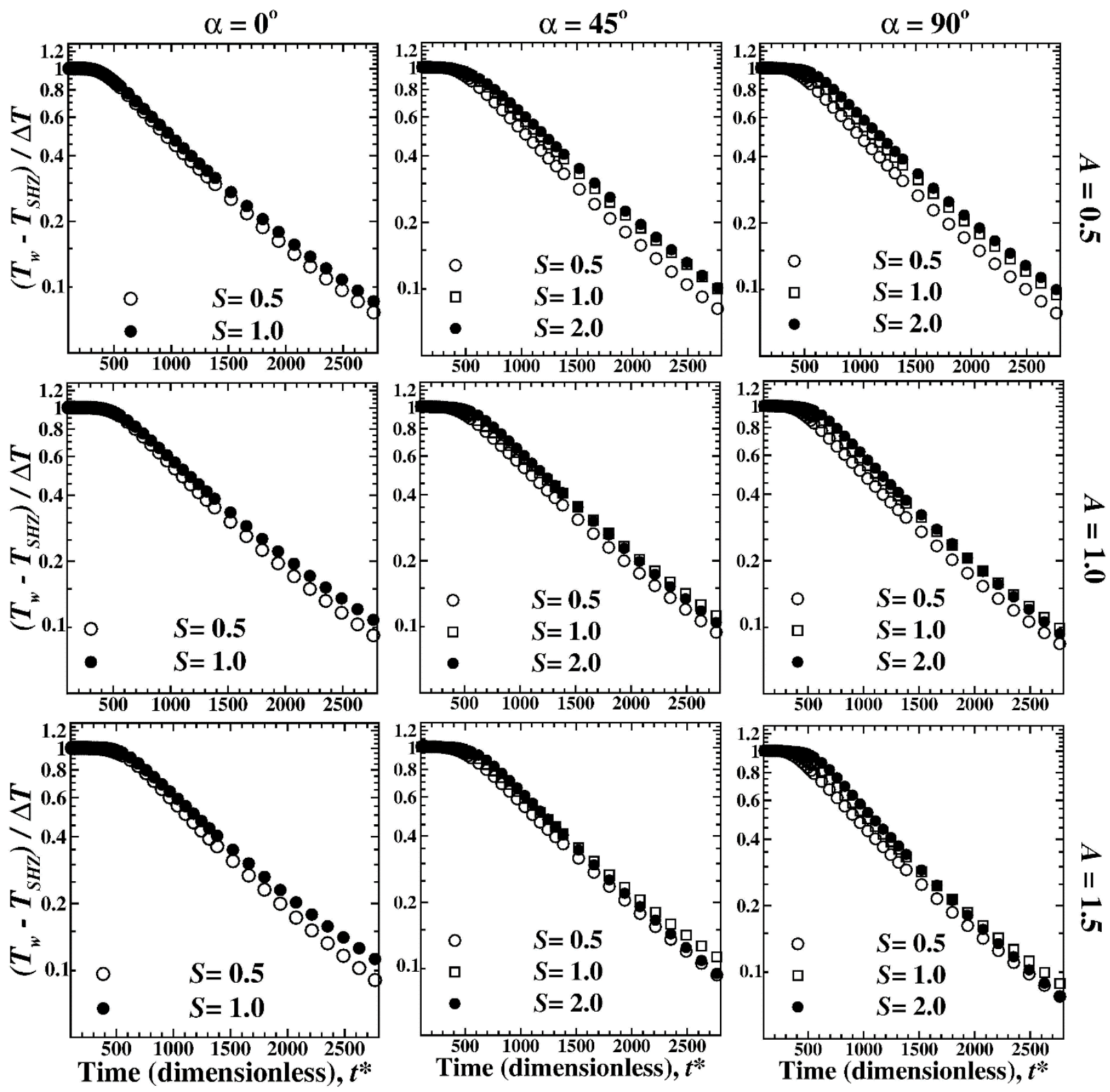

Figure 12. Evolution of Slowest Heating Zone (SHZ) temperature with time for different cases. 
(a)

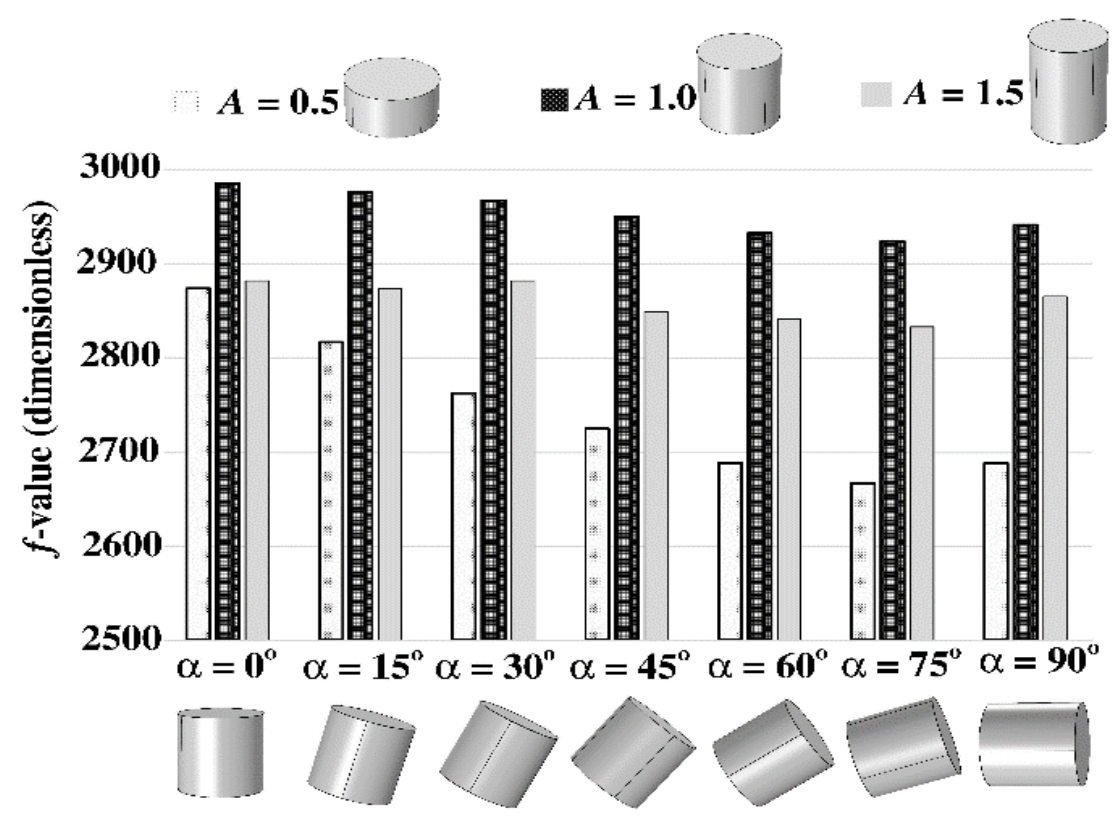

(b)

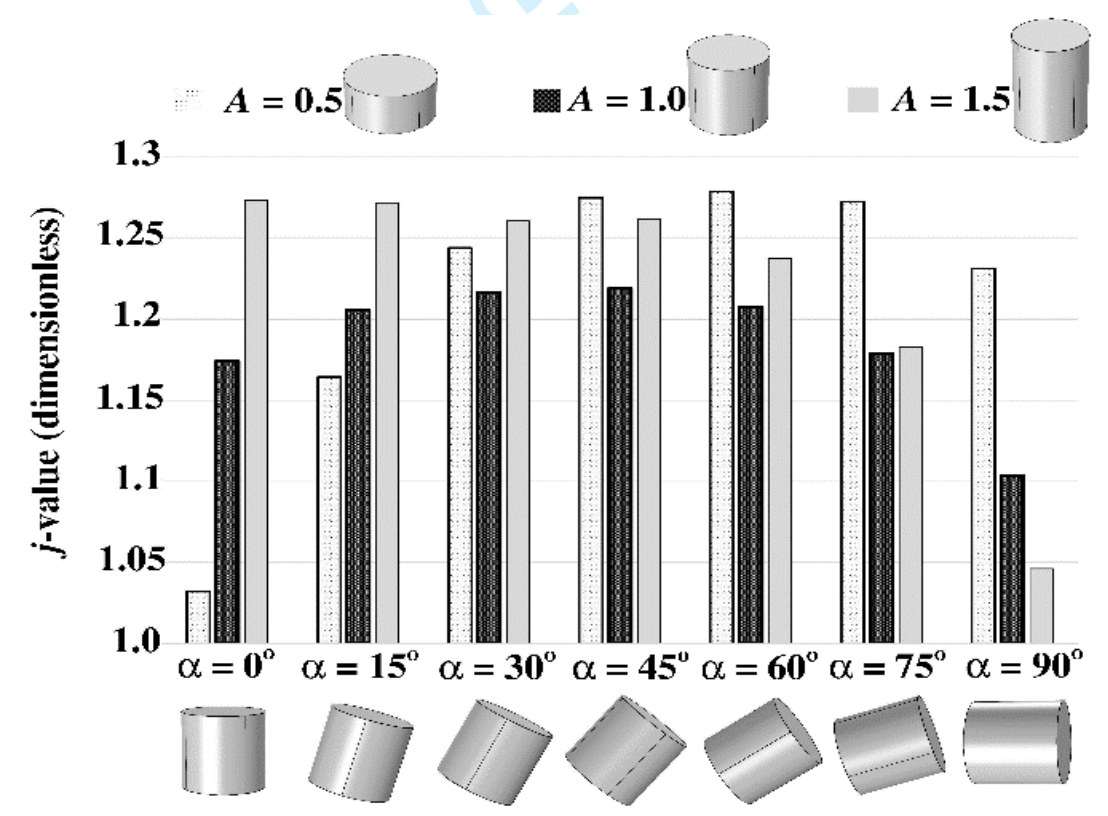

Figure 13. Effect of orientation of cylindrical cans on $f$-value and $j$-value. 
(a)

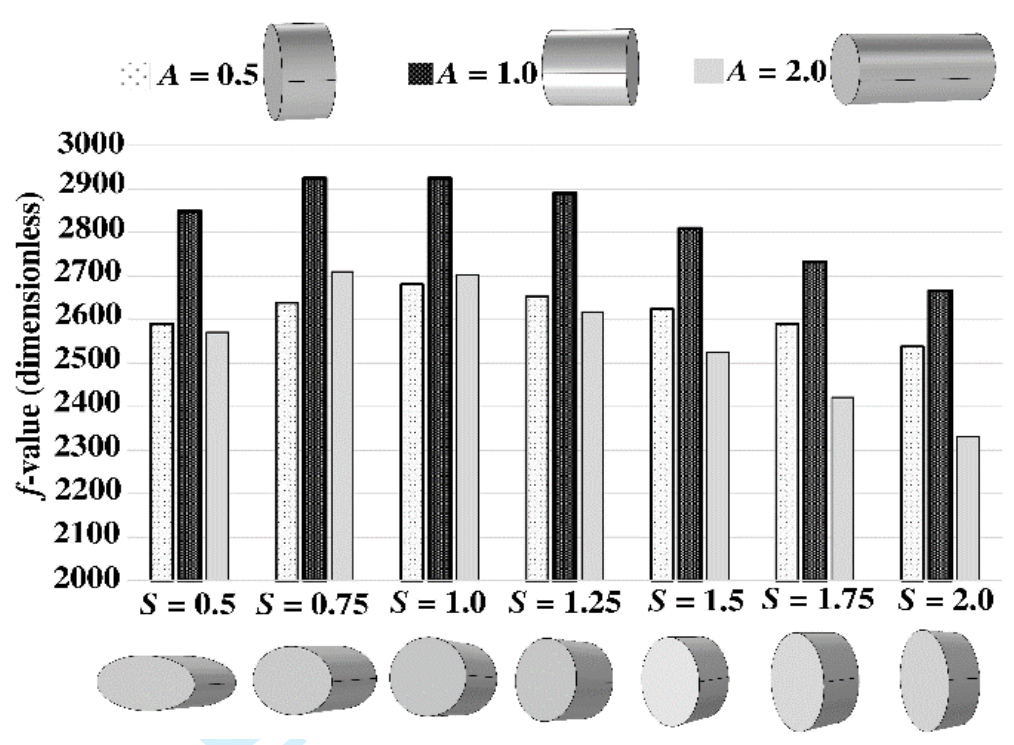

(b)

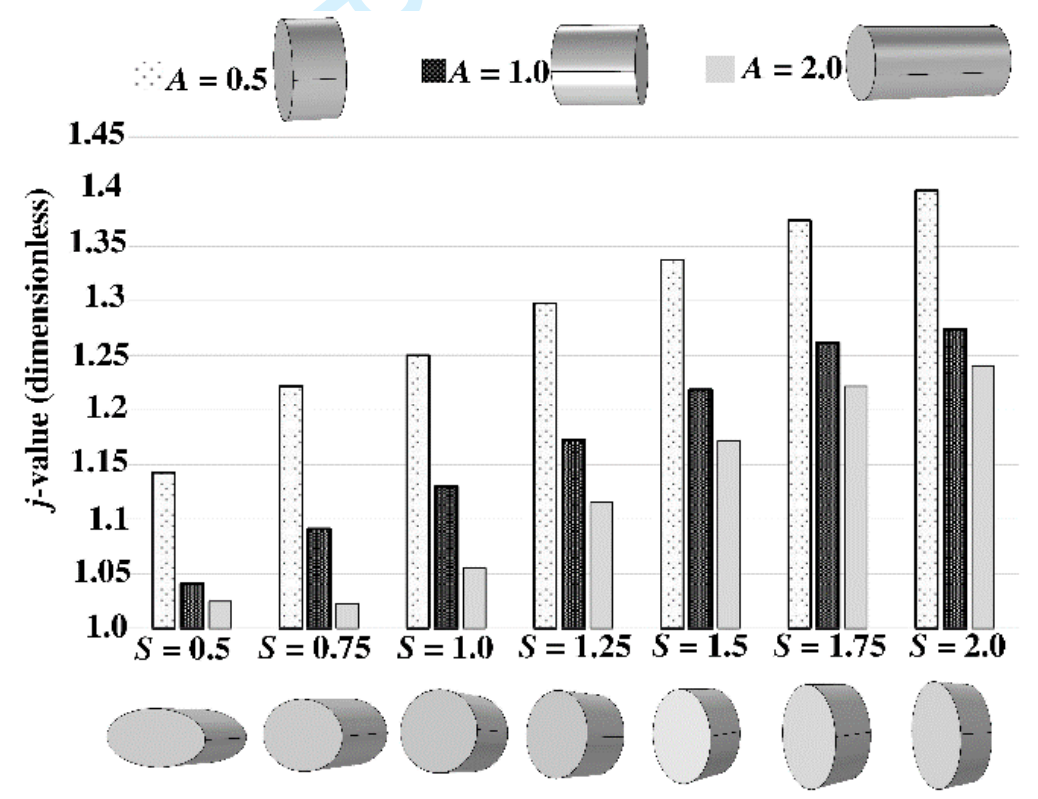

Figure 14. Effect of $S$ of horizontal cans on $f$-value and $j$-value.

(a) 


$$
A=0.5
$$

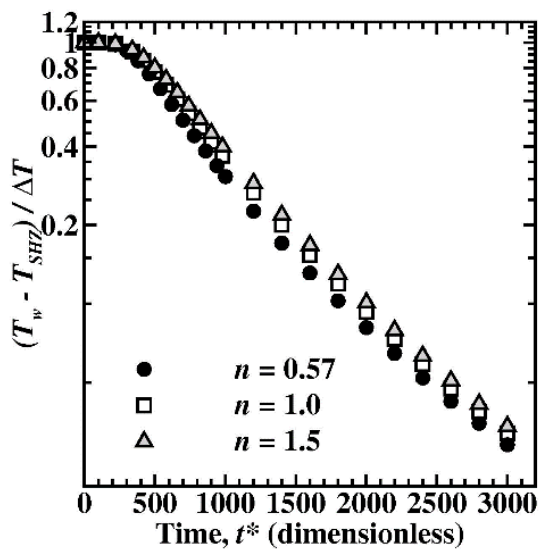

$A=1.0$

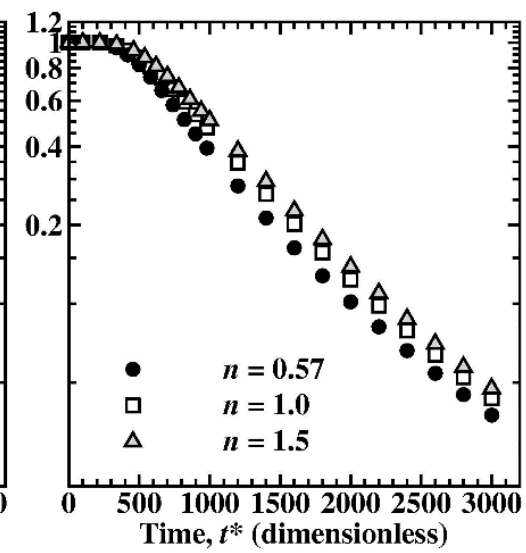

$A=\mathbf{1 . 5}$

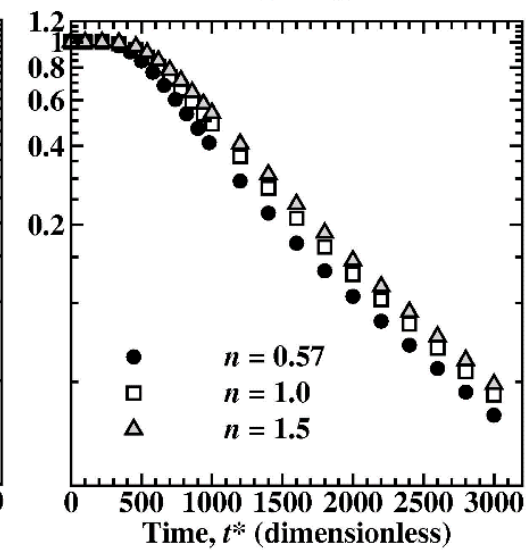

(b)
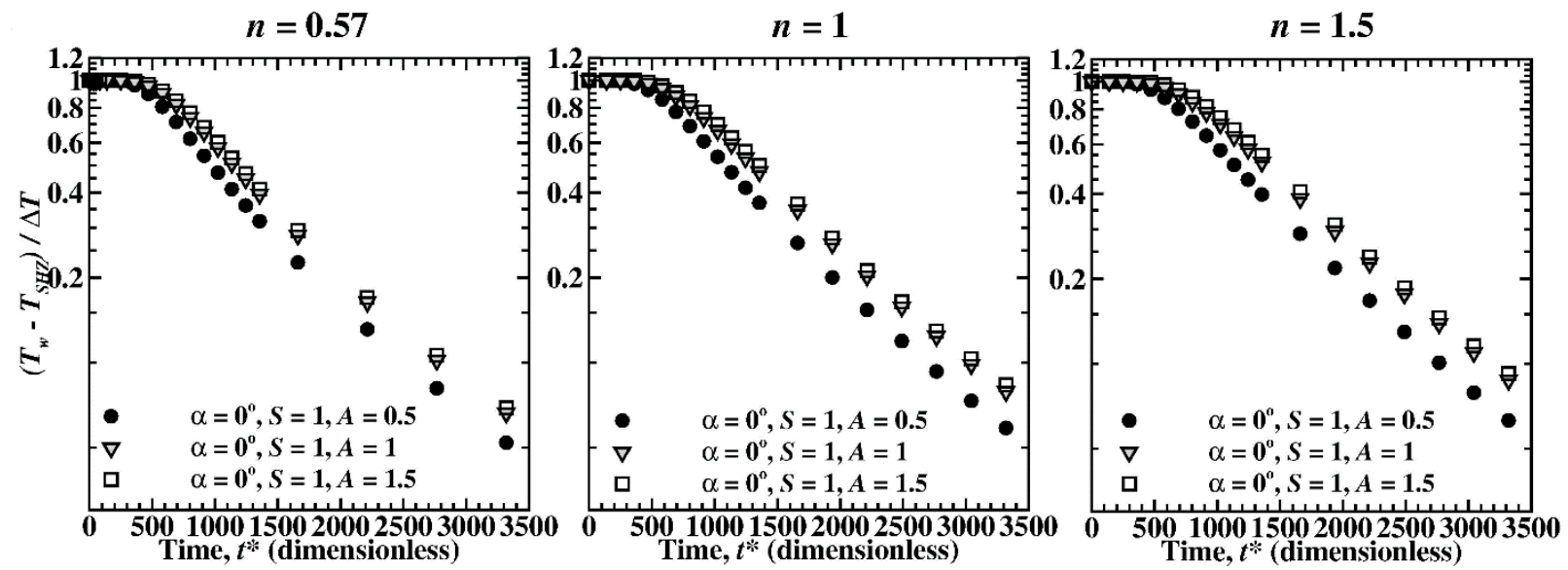

(c)
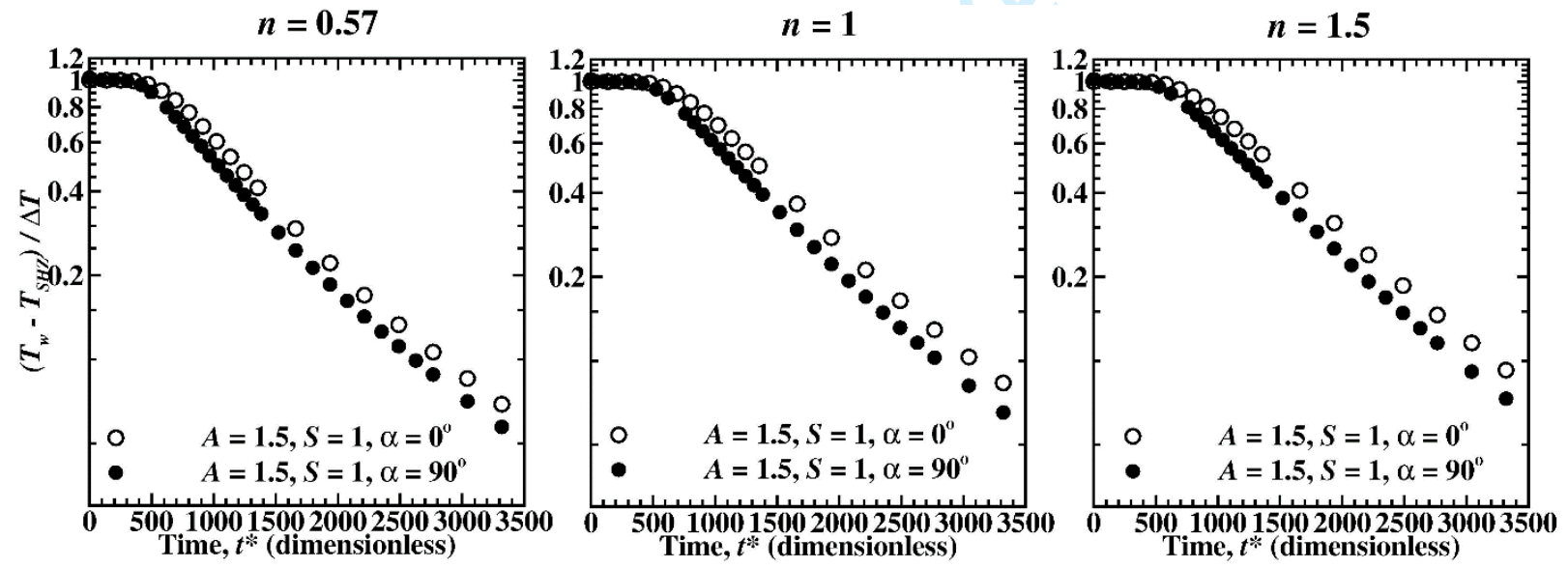

Figure 15. Effect of rheology (power law index $n=0.57, n=1, n=1.5$ ) on heat penetration for constant Prandtl number, $\operatorname{Pr}=79481$. 


\section{RESPONSE TO REVIEWERS' COMMENTS (JFPE-2019-Jan-0015.R1)}

We are grateful to the reviewers for their valuable inputs and suggestions for improving our work. Regarding the role of CFD in these studies and the implementation of experimental results, we plan to include these as our future work. This study serves as an initial platform towards the formulation of these multi-parameter models for 3-D geometries with natural convection encompassing non-Newtonian fluids. In response to the reviewer's comments, the manuscript has been revised as follows:

\section{$\underline{\text { Reviewer \# } 1}$}

This reviewer has not made any suggestions requiring changes in the manuscript. We are thankful for the acceptance of the manuscript.

\section{$\underline{\text { Reviewer \# } 2}$}

\section{Axes are defined in meters in nomenclature but figures are shown with non-graduated - unmarked} axes. Better to put distance values on axes or provide scale.

The formulation of the problem is not entirely in its dimensionless form as it is important to give an idea about the length scales of the problem and COMSOL also solves the system of equations in a dimensional form. However, the results are reported in a dimensionless format to enhance their applicability irrespective of the length scale for a given set of dimensionless parameters. For instance, the spatial coordinates are in meter but the spatial variation shown in Figures 2 and 3 is plotted against $\mathrm{x} / H$. In Figure 2, the variation of the Nusselt number along the length of the can is shown for "short" and "tall" cans. Since the lengths are different in the two cases, it is more convenient to scale the distance with the total height/length for comparison. The non-graduated cylinders qualitatively aid our understanding of the effects of orientation and aspect ratio of the cylinder. Besides, Nusselt number is plotted on the y-axis which is a dimensionless parameter, it is also helpful for the consistency to keep the length dimensionless. Some new text has been added on page 12 to address this issue. 


\section{Literature review covers very old literature and some are superfluous representing currently well-} known general information. It may be considerably condensed to represent current trends. The introduction section has been revised to address this issue. On this count, changes are made on pages $5,6,26$ and 27.

\section{Justify current use of equivalent diameter for elliptical cross-section. Usually in Chemical} Engineering, it is based on hydraulic radius. Was a similar calculation done here? Can geometric mean of the two length scales qualify for equivalent diameter? I find this somewhat misleading and prone to wrongful replication in subsequent work, should this work be accepted.

While the concept of hydraulic diameter is commonly used in modifying the Moody diagram for estimating the friction factor, $\mathrm{D}_{e q}$ is simply defined in this work to be able to compare different cross sections for the same can height/length. The aspect ratio $A$ is an indicative of how long (or height) the can is and the aspect ratio $S$ is indicative of the cross sectional area $(S=1$ for cylindrical and $S=0.5$ for elliptical).

For an elliptical can, $\mathrm{D}_{e q}$ is the diameter of the equivalent circle of equal area:

Mathematically, $\pi \times \mathrm{D}_{e q}{ }^{2} / 4=\pi \mathrm{ab}$ or $\mathrm{D}_{e q}=\sqrt{ }(4 \mathrm{ab})$

For an elliptical shape, it is $D_{h}=\frac{2 \sqrt{2} a b}{\sqrt{a^{2}+b^{2}}}$ which can easily be converted into $D_{e q}$ or vice-a-versa and therefore, the two measures $D_{e q}$ and $D_{h}$ are related only by a constant factor which is a function of $a / b$ only. Some new text has now been added on page 7 to address this issue.

4. Why not scale $S$ also with major axis length to equivalent diameter? Even here in the circular case will reduce to 1 and be consistent with vertical aspect ratio $L / D_{e q}$.

The aspect ratios $S$ and $A$ indicate two separate geometrical features of the can, namely, shape of the crosssectional area and the height of the can. Thus, any definition without interdependence will be fine. As suggested, $A=H / D_{e q}$ and $S=a / D_{e q}$ could also be suitable definitions to treat these parameters independently. So, it is just a matter of choice. However, this possibility has been indicated on page 7 . 


\section{Why should initial temperature be at $40^{\circ} \mathrm{C}$ ? Probably these stuffs are refrigerated or cold storaged prior to thermal sterilization. The given initial temperature is odd.}

The value of the initial temperature is indeed arbitrary as the food- stuff under consideration can be subjected to different pre-processing conditions. However, bearing in mind the power of dimensional considerations, the resent results are equally valid for any other value of the initial temperature as long as the dimensionless temperature is same in the two applications. Keeping the various possibilities of the initial conditions in mind, the results are presented in a dimensionless form for the aforementioned ranges of dimensionless groups. Some previous studies have also considered $40^{\circ} \mathrm{C}$ as the initial temperature assuming that the food is at room temperature so we have also used this value in our work.

Some new text has been added on page 23 .

\section{Granted that a uniform temperature boundary condition was applied on all walls in previous} literature, but how realistic are these? What for e.g. is the presence of vapor space which is likely to exist on the surface of the liquid in the can? What does previous literature say about this? I would expect the air space to act as an insulator and considerably reduce the heat transfer rate.

The uniform temperature condition is a realistic assumption as saturated steam at $1 \mathrm{~kg}$ pressure is commonly used for heating. Thus, the can walls are nearly maintained at the condensation temperature corresponding to this pressure.

The effect of the vapor space is not taken into account(i) due to the fact that the top surface does not contribute to the free convection (Figure 9); (ii)in case there is a vapor space which will add another thermal resistance to the overall resistance to heat transfer. In this case, the results reported herein represent a lower bound on the heating time needed. Thus, the presence of some vapor space at the top will not alter the trends reported in this work appreciably. Indeed, this assertion is supported by the experimental results of Mohamed (2007... New reference now added).

The experimental results of Mohamed (2007) show that for $50 \mathrm{~mm}$ tall can of $70 \mathrm{~mm}$ diameter, varying the head space from $5 \mathrm{~mm}$ to $10 \mathrm{~mm}$ changes the heat transfer coefficient from $54.2 \mathrm{~W} / \mathrm{m}^{2 \circ} \mathrm{C}$ to $50.8 \mathrm{~W} / \mathrm{m}^{2 \circ} \mathrm{C}$, 
i.e., by $8 \%$ or so which is well within the uncertainty of their results. Thus, they reported an overall average heat transfer coefficient value for the range of headspace investigated as $52.73 \pm 1.75 \mathrm{~W} / \mathrm{m}^{2}{ }^{\circ} \mathrm{C}$. So this effect is indeed small. On the other hand, the inclusion of vapor space would entail consideration of multiphase heat transfer and phase equilibrium etc. which will add to the complexity of CFD in such system.

However, this is a good point and we have added some text on page 23 .

\section{Why not include temperature dependence on $\mathrm{Cp}$ and $\mathrm{k}$ ? Can a sensitivity analysis be carried out?}

Unfortunately, the reliable data for the temperature dependence of $C_{p}$ and $k$ for the model fluid of this specific CMC concentration is not available in the literature to the best of our knowledge. Since this study is intended towards the delineation of the effect of geometrical and rheological characteristics of the cans and food, respectively, the role of temperature dependence of $C_{p}$ and $k$ was not considered. However, in order to assess the possible effect of this assumption, additional eight simulations were carried out by varying the values of $C_{p}$ and $k$ by $\pm 5 \%$ from their mean values. The resulting values were uniformly scattered by up to $2 \%$ around the results obtained with the constant values of $C_{p}$ and $k$, as shown below. Therefore, this effect seems to be minor and this figure is not included in our revised manuscript. However, some new text has been added on page 23 .

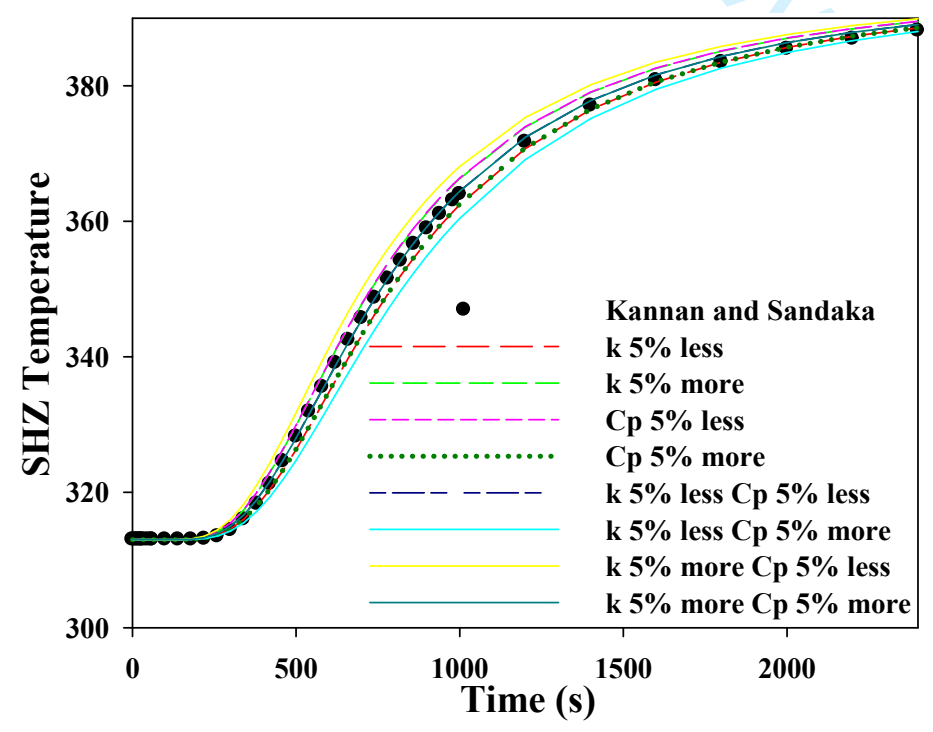




\section{Font sizes of some symbols for e.g. $L$ are inconsistent. Please check.}

The text has been corrected as suggested.

\section{Give further justification for Eqn. 12 as Ea is already scaled with RT. Given connection to} combustion appears to be irrelevant here.

In Equation 12, the activation energy, $E_{a}$ is scaled using $R T_{a v g}$. It simply means that the $T$ is a harmonic mean of the temperature limits of the process. This term is obtained while rendering the momentum equations dimensionless. When the exponential function of the temperature dependent viscosity is linearized using the Taylor series expansion and the temperature is scaled by the temperature difference, the dimensionless group $\zeta=\frac{E_{a}}{R T_{w}}\left(\frac{T_{w}-T_{o}}{T_{o}}\right)$ is obtained which is an indicative of the temperature sensitivity of the viscosity. However, the sentence concerning combustion has now been removed to safe guard against this potential confusion. Some additional text has been inserted on page 10 .

\section{I do not see system becoming sluggish with time (relevant figures 3 and 4). They seem having a constant rate even finally in the time interval considered.}

By the term 'sluggishness', we simply mean the rate of change of temperature. In the first plot in Figure 4, the rate of change of volume average temperature is continually decreasing. Text is modified on Page 12.

\section{Not clear if initial time steps in one scheme are in the order of milli seconds, how time steps of 0.05 and 0.1 seconds also become acceptable?}

As correctly pointed out by the reviewer, different time steps do not give exactly the same numerical results, but these differ only in second/third decimal places. Thus, the differences between the results obtained using time steps of 0.05 and 0.1 second are too small to be visible on the plots. In general, the time-scale of the process is considerably large (more than 2500 seconds). So these time steps are very small for a process in this time-scale despite the fast initial heating rate. The size of the time step can be reduced to the order 
of milliseconds which is inevitably accompanied by large computational time needed. Also, the choice of the step size depends on the robustness of the solver algorithm.

Some text has been added on page 12.

12. Fig. 4: What would the authors like to conclude here? Are the flows and temperature profiles corresponding to axisymmetric conditions or not?

The literature results used here for validation were obtained for the 2D axisymmetric conditions. Since the present work takes into account different configurations where axi-symmetry assumption does not hold under all conditions of inclination, the 3-D energy and momentum equations were solved. Thus, Figure 4 simply verifies that, in the laminar regime, the results obtained by the $3 \mathrm{D}$ computations and $2 \mathrm{D}$ axisymmetric conditions are in agreement. This is a validation step along with grid and time step tests to ensure that the present results are free from numerical artefacts.

Some new text has been added on page 14 to address this issue.

\author{
13. It is well known to an engineer and scientists that the length of the velocity vector is proportional \\ to its magnitude. \\ The text emphasizing this point has been removed due to its redundancy.
}

\title{
14. It appears that the authors have not provided much discussion on effect of inclination on velocity plots and hence requires more elaboration. The inclination is also probably difficult to implement in a practical scale.
}

In response to this comment, the text is somewhat modified to emphasize this point. In general, partial inclination does not seem to enhance the convective flow as significantly as in the case of horizontal orientation. Thus, the two extremes, vertical and horizontal orientations have been given more importance in the discussion. Inclination may be difficult to implement if we have to consider the rotation of the retort 
16. Also how did the authors define the cut plane in inclined flows? Why would it be representative of the flow inside the food can?

Defining the cut planes for inclined cans is indeed problematic as the axial symmetry does not hold. WE have added some new text (on page 14) to explain what exactly has been done here. Some new text has been added on page 14 .

\section{Equation 15 and Table 2: Parameter $d$ in the model consistently has very low values and seems} statistically insignificant. For this and other parameters a statistical significance test is suggested and superfluous parameters be removed.

The empiricism in Equation 15 is based on the combination of an exponential decay function and a Gaussian-like function. The parameter $d$ is multiplied with $t^{2}$, thus it has a balancing effect on the function. Despite its small value, the exclusion of this term leads to poor fitting as it is a multiplication factor. Preliminary analysis indicated this to be significant effect. No change has been made on this count.

\section{How will the correlation given by Equation 15 be useful?}

Since Nusselt number indicates the relative strength of convection and conduction heat transfer, modelling Nusselt number throughout the process describes the evolution of these two heat transfer regimes. These 
correlations can be used at different length scales given that the dimensional parameters are matched to predict the heat transfer regimes and estimate the heat transfer coefficient for a given application.

Some new text has been added on page 17.

\section{Section 5.6: Is SHZ located at one unique location or more than one? Explain "A simple definition of the sterilization time is the time required for the SHZ temperature to reach the threshold temperature everywhere in the can and this may of course vary from one case to another"}

SHZ is the coldest point in the can. For the simple can geometries considered in this work, the SHZ is not found at multiple locations. However, it is worth noting here that the SHZ is not always a single point. For a vertical cylindrical can, the SHZ is found at a radial position between the axis and the wall close to the bottom. Considering the axial symmetry, these points constitute a ring. The text has been modified on pages (17-18) to clarify this point.

Since SHZ is the coldest point, for a certain temperature of the SHZ, the temperature everywhere else in the can is higher. If one needs to ensure that the entire food in the can is heated above a certain temperature to kill the germs, the SHZ temperature should reach at least that specific temperature. Thus, the heating characteristics of the SHZ alone can be used to define parameters determining various aspects of the sterilization process. The discussion regarding sterilization time starts with a simplistic definition used previously in literature (Varma \& Kannan, 2005) that assumes that if the SHZ reaches the threshold temperature, the sterilization is complete. This is a probably an over simplification which is useful for a crude comparison. The inadequacy of this definition is pointed out in the discussion where statistical parameters $f$-and $j$-values were introduced which provide a clearer representation of the heating dynamics. However, while discussing duration, efficiency and production planning of batch process, sterilization time offers a convenient indicator to use.

20. Section 5.6: Heading is misleading as rotation may also mean can rotation on which a study had already been published. 
2

3

4

5

6

7

8

9

10

In response to this comment, the text in the heading has been corrected on page 18 .

\section{Section 5.7: Define $\Delta T$ clearly- Is Numerator term also $T_{w}-T_{S H Z}$ ?}

Yes. $T_{S H Z}$ is a variable, while $T_{w}$ and $T_{o}$ are constant boundary and initial conditions. $T_{w}-T_{S H Z}$ is the temperature difference between the hot wall and the temperature of the coldest point inside the can. The value of $T_{S H Z}$ continually decreases with time. $\Delta T$ is the temperature difference between the temperature limits, $T_{w}$ and $T_{o}$ and is used to scale this difference. In response to the comment, the text has been modified clarifying this point further, please see page 20. 


\section{GRAPHICAL ABSTRACT}
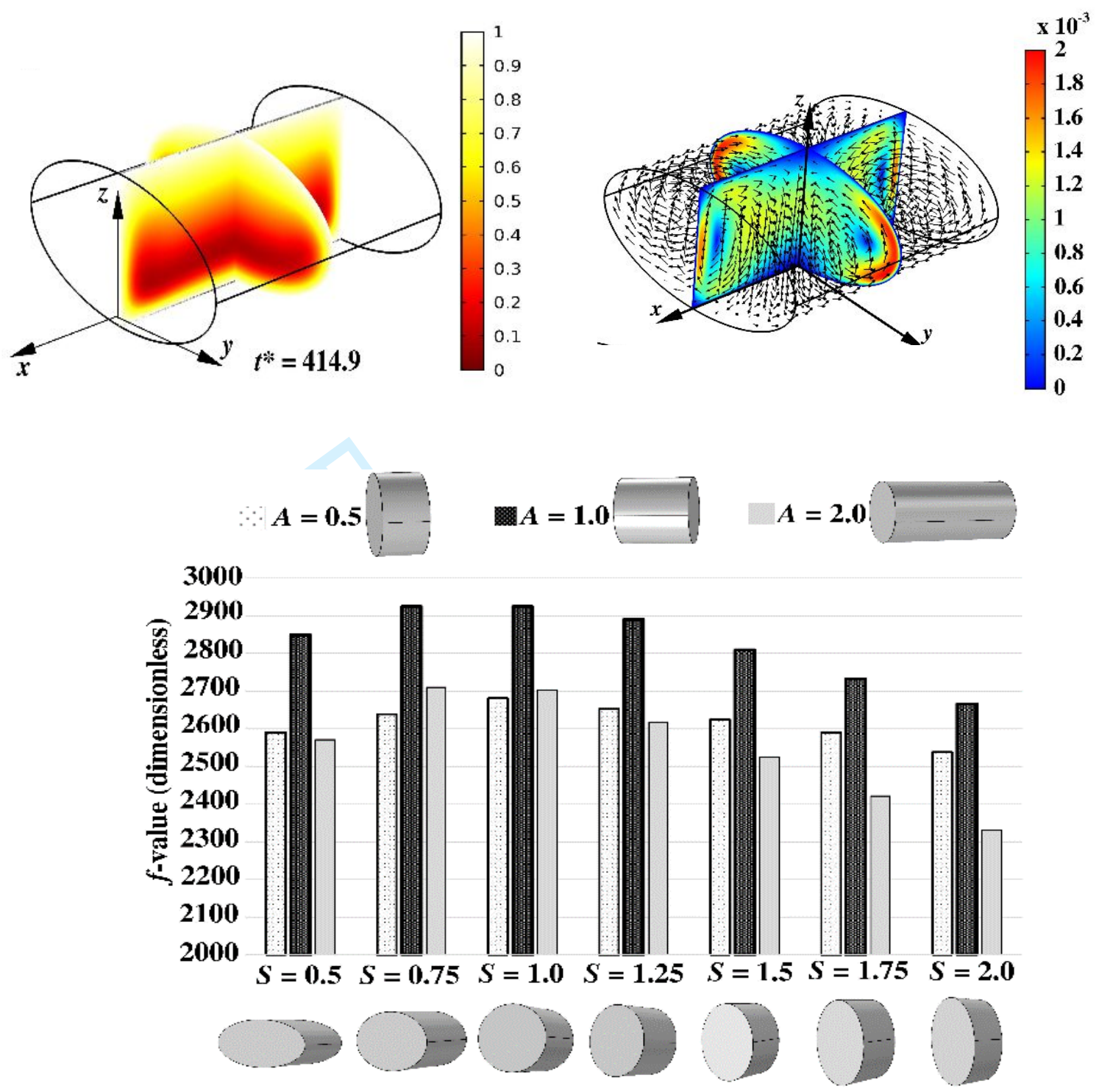